Supporting Information

\title{
11-Step and Scalable Total Synthesis of Hamigeran M Enabled by Five C-H Functionalizations
}

\author{
Baiyang Jiang, and Mingji Dai* \\ Department of Chemistry and Center for Cancer Research \\ Purdue University, 720 Clinic Drive, West Lafayette, IN 47907 (USA)
}




\section{Table of Contents}

Part 1. Experimental procedures and spectra data............................................S3

Part 2. X-ray structure and analysis data...............................................................S22

Part 3. References.............................................................................................................524

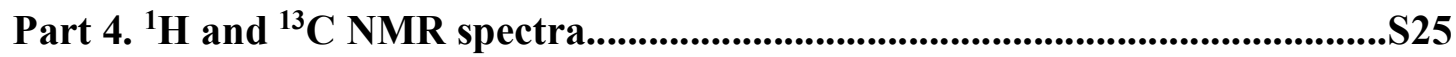




\section{Part 1. Experimental procedures and spectra data.}

General Methods. NMR spectra were recorded on Bruker spectrometers $\left({ }^{1} \mathrm{H}\right.$ at $400 \mathrm{MHz}$,

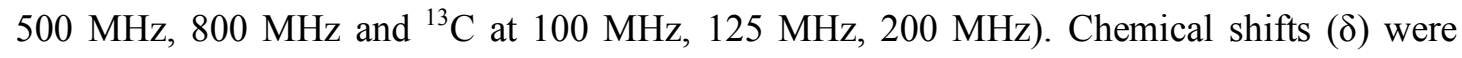
given in ppm with reference to solvent signals $\left[{ }^{1} \mathrm{H} \mathrm{NMR}: \mathrm{CHCl}_{3}(7.26) ;{ }^{13} \mathrm{C} \mathrm{NMR}: \mathrm{CDCl}_{3}\right.$ (77.2), $\mathrm{C}_{6} \mathrm{D}_{6}$ (128.02), $\mathrm{CD}_{3} \mathrm{OD}$ (49.0)]. High-resolution mass measurements for compound characterization were carried out using a Waters SYNAPT G2-Si system with QuanTof analyzer or an Agilent 6550 QTOF system. Column chromatography was performed on silica gel. All reactions requiring heating were heated in an oil bath. All reactions sensitive to air or moisture were conducted under argon atmosphere in dry and freshly distilled solvents under anhydrous conditions, unless otherwise noted. Anhydrous THF and toluene were distilled over sodium benzophenone ketyl under Argon. Anhydrous $\mathrm{CH}_{2} \mathrm{Cl}_{2}$ was distilled over calcium hydride under Argon. All other solvents and reagents were used as obtained from commercial sources without further purification. The NMR yield was determined using $\mathrm{BrCH}_{2} \mathrm{CH}_{2} \mathrm{Br}(0.5$ equiv.) as internal standard. Room temperature is about $23{ }^{\circ} \mathrm{C}$.

\section{Experiment procedure}

Compound $\mathbf{2 6}^{[1]}, \mathbf{3 0}^{[2]}, \mathrm{TMPZnCl} \cdot \mathrm{LiCl}^{[3]}$ were prepared according to reported procedures.

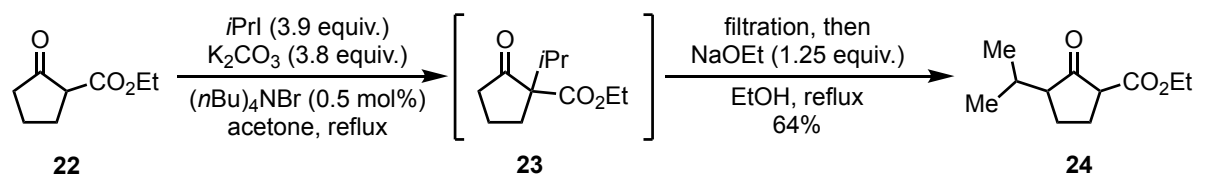

Compound 24 was prepared through modification of the reported method. ${ }^{[4]}$ To a $100 \mathrm{~mL}$ flask fitted with condenser were added 22 (4.10 g, $26.3 \mathrm{mmol}, 1.0$ equiv.), potassium carbonate (10.25 g, $74.2 \mathrm{mmol}, 2.8$ equiv.), $i \operatorname{PrI}$ (7.7 mL, $77 \mathrm{mmol}, 2.9$ equiv.), TBAB (40 mg, $0.12 \mathrm{mmol}, 0.5 \mathrm{~mol} \%)$ and acetone $(6 \mathrm{~mL})$. The reaction was stirred at reflux for $23 \mathrm{~h}$. Then more potassium carbonate $(3.5 \mathrm{~g}, 25.3 \mathrm{mmol}, 1.0$ equiv.) and $i \operatorname{PrI}(2.6 \mathrm{~mL}, 26 \mathrm{mmol}, 1.0$ equiv.) were added. The reaction was further stirred at reflux for $24 \mathrm{~h}$, then cooled down to room temperature. The solid was removed by filtration and the filtrate was concentrated and azeotroped with toluene $(10 \mathrm{~mL})$ twice. To the residue was added $\mathrm{NaOEt}$ in EtOH $(25 \mathrm{~mL}$, prepared by adding $0.75 \mathrm{~g}(32.7 \mathrm{mmol})$ of $\mathrm{Na}$ to $25 \mathrm{~mL}$ of $\mathrm{EtOH}, 1.25$ equiv.). The mixture 
was stirred at reflux for $7.5 \mathrm{~h}$ before toluene $(10 \mathrm{~mL})$ was added. The mixture was heated to remove EtOH until $\sim 12 \mathrm{~mL}$ of solution was left. The reaction was then quenched with saturated aqueous ammonium chloride $(20 \mathrm{~mL})$ and extracted with ether $(20 \mathrm{~mL} \times 3)$. The combined organic phase was washed with brine, dried $\left(\mathrm{Na}_{2} \mathrm{SO}_{4}\right)$, filtered and concentrated. The residue was purified on column chromatography (hexane/EtOAc $=100 /$ to $88 / 12$ ). Compound 24 was obtained as a light pink oil (3.34 g, 64\%). The characterization data was in accordance with previous report. ${ }^{[4]}$

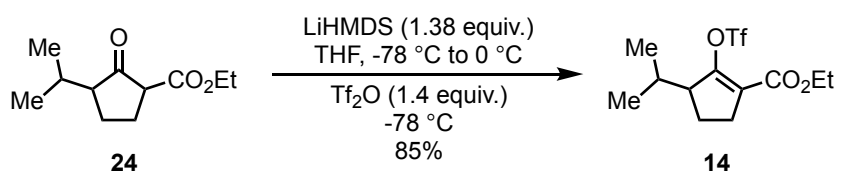

To $24(3.34 \mathrm{~g}, 16.9 \mathrm{mmol})$ in THF $(80 \mathrm{~mL})$ at $-78{ }^{\circ} \mathrm{C}$ was added LiHMDS $(23.3 \mathrm{~mL}, 1 \mathrm{M}$ in THF, $23.3 \mathrm{mmol}, 1.38$ equiv.). The mixture was warmed up to $0{ }^{\circ} \mathrm{C}$ and stirred for $2 \mathrm{~h}$. Then the reaction was cooled down to $-78^{\circ} \mathrm{C}$, and triflic anhydride $(4.0 \mathrm{~mL}, 23.6 \mathrm{mmol}, 1.40$ equiv.) was added dropwise. The resulting mixture was stirred at this temperature for $3 \mathrm{~h}$ before it was quenched with saturated aqueous ammonium chloride $(20 \mathrm{~mL})$ and extracted with ether $(20 \mathrm{~mL} \times 3)$. The combined organic phase was washed with brine, dried $\left(\mathrm{Na}_{2} \mathrm{SO}_{4}\right)$, filtered and concentrated. The residue was purified on column chromatography (hexane/EtOAc $=95 / 5$ to 74/26). Compound 14 was obtained as colorless oil (4.72 g, 85\%). ${ }^{1}$ H NMR (500 MHz, $\left.\mathbf{C D C l}_{3}\right) \delta 4.27(\mathrm{q}, J=7.1 \mathrm{~Hz}, 2 \mathrm{H}), 3.01-2.92(\mathrm{~m}, 1 \mathrm{H}), 2.70-2.55(\mathrm{~m}, 2 \mathrm{H}), 2.09-1.95(\mathrm{~m}$, 2H), $1.80-1.69(\mathrm{~m}, 1 \mathrm{H}), 1.32(\mathrm{t}, J=7.1 \mathrm{~Hz}, 3 \mathrm{H}), 0.95$ (d, $J=6.9 \mathrm{~Hz}, 3 \mathrm{H}), 0.86(\mathrm{~d}, J=6.8$ $\mathrm{Hz}, 3 \mathrm{H}) ;{ }^{13} \mathbf{C}$ NMR (125 MHz, $\left.\mathbf{C D C l}_{3}\right) \delta 162.5,155.3,124.1,118.4$ (q, $\left.J=319 \mathrm{~Hz}\right), 61.2$, 50.6, 28.6, 28.2, 20.3, 19.9, 16.7, 14.0; ${ }^{19}$ F NMR (471 $\mathbf{M H z}, \mathbf{C D C l}_{3}$ ) $\delta$-75.85; IR (film): 2965, 1721, 1427, 1206, 1140, 1126, 839, $604 \mathrm{~cm}^{-1}$; HRMS (ESI): $\mathrm{m} / z$ Calc. for $\mathrm{C}_{12} \mathrm{H}_{18} \mathrm{~F}_{3} \mathrm{O}_{5} \mathrm{~S}[\mathrm{M}+\mathrm{H}]^{+}: 331.0821$, found: 331.0820 .

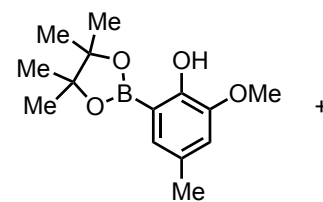

26

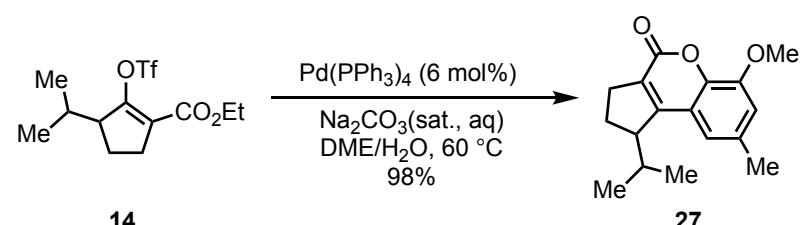

14

To 26 (4.1 g, 12.4 mmol, 1.0 equiv.), 14 (4.0 g, 13.3 mmol, 1.1 equiv.) and $\mathrm{Pd}_{(}\left(\mathrm{PPh}_{3}\right)_{4}(0.87 \mathrm{~g}$, 
$0.75 \mathrm{mmol}, 6.0 \mathrm{~mol} \%)$ were added degassed DME $(70 \mathrm{~mL})$ and degassed saturated aqueous sodium carbonate $(25 \mathrm{~mL})$. The reaction was warmed up to $60{ }^{\circ} \mathrm{C}$ and stirred for $100 \mathrm{~min}$. Then the mixture was cooled down to room temperature and quenched with saturated aqueous ammonium chloride $(40 \mathrm{~mL})$. The residue was extracted with ether $(40 \mathrm{~mL} \times 3)$. The combined organic phase was washed with brine, dried $\left(\mathrm{Na}_{2} \mathrm{SO}_{4}\right)$, filtered and concentrated. The residue was purified by column chromatography (hexane/EtOAc $=10 / 1$ to $6 / 1$ ). Compound 27 was obtained as a light-yellow solid (3.23 g, 98\%). mp 139-140 ${ }^{\circ} \mathrm{C} ;{ }^{1} \mathbf{H}$ NMR $\left(\mathbf{5 0 0} \mathbf{~ M H z}, \mathbf{C D C l}_{3}\right) \delta 6.88(\mathrm{~s}, 1 \mathrm{H}), 6.84(\mathrm{~s}, 1 \mathrm{H}), 3.93(\mathrm{~s}, 3 \mathrm{H}), 3.49-3.41(\mathrm{~m}, 1 \mathrm{H}), 2.89-2.73$ $(\mathrm{m}, 2 \mathrm{H}), 2.40(\mathrm{~s}, 3 \mathrm{H}), 2.38-2.28(\mathrm{~m}, 1 \mathrm{H}), 2.14-2.05(\mathrm{~m}, 2 \mathrm{H}), 1.10(\mathrm{~d}, J=6.9 \mathrm{~Hz}, 3 \mathrm{H}), 0.69$ $(\mathrm{d}, J=6.8 \mathrm{~Hz}, 3 \mathrm{H}) ;{ }^{13} \mathbf{C}$ NMR (125 MHz, $\left.\mathbf{C D C l}_{3}\right) \delta 159.9,157.8,147.2,142.3,133.7,128.2$, 118.7, 116.0, 113.8, 56.2, 51.4, 30.7, 30.5, 23.5, 22.0, 21.6, 16.2; IR (film): 2957, 1715, 1583, 1466, 1392, 1146, $1043 \mathrm{~cm}^{-1}$; HRMS (ESI): $\mathrm{m} / z$ Calc. for $\mathrm{C}_{17} \mathrm{H}_{21} \mathrm{O}_{3}[\mathrm{M}+\mathrm{H}]^{+}:$273.1485, found: 273.1484 .
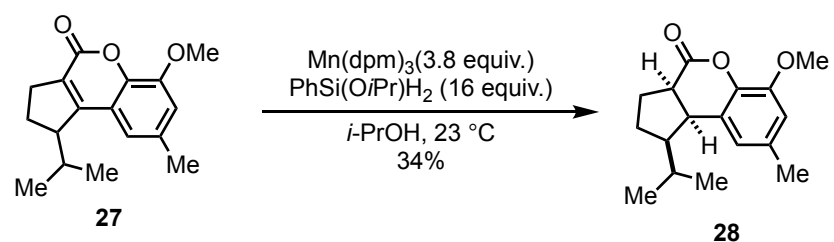

For the $\mathrm{Mn}(\mathrm{dpm})_{3}$ procedure: To $27(504.3 \mathrm{mg}, 1.85 \mathrm{mmol}, 1.0$ equiv.) in a flame-dried flask was added $i \mathrm{PrOH}(60 \mathrm{~mL})$ in the glovebox. Then $\mathrm{Mn}(\mathrm{dpm})_{3}(2.46 \mathrm{~g}, 4.07 \mathrm{mmol}, 2.2$ equiv.) and $\mathrm{PhSiH}_{2}(\mathrm{O} i \mathrm{Pr})(1.65 \mathrm{~mL}, 9.2 \mathrm{mmol}, 5.0$ equiv.) were added and the resulting mixture was stirred at room temperature for $2 \mathrm{~h}$. Then more $\mathrm{PhSiH}_{2}(\mathrm{O} i \mathrm{Pr})(1.0 \mathrm{~mL}, 5.6 \mathrm{mmol}, 3.0$ equiv. $)$ was added and the mixture was stirred for $2 \mathrm{~h}$. Then more $\mathrm{Mn}(\mathrm{dpm})_{3}(0.90 \mathrm{~g}, 1.5 \mathrm{mmol}, 0.8$ equiv.) and $\mathrm{PhSiH}_{2}(\mathrm{O} i \mathrm{Pr})(1.0 \mathrm{~mL}, 5.6 \mathrm{mmol}, 3.0$ equiv. $)$ were added and the mixture was stirred for $15 \mathrm{~h}$. Then more $\mathrm{Mn}(\mathrm{dpm})_{3}(0.90 \mathrm{~g}, 1.5 \mathrm{mmol}, 0.8$ equiv. $)$ and $\mathrm{PhSiH}_{2}(\mathrm{O} i \mathrm{Pr})(1.0$ $\mathrm{mL}, 5.6 \mathrm{mmol}, 3.0$ equiv.) were added and the mixture was stirred for $2 \mathrm{~h}$. Then more $\mathrm{PhSiH}_{2}(\mathrm{O} i \mathrm{Pr})(0.67 \mathrm{~mL}, 1.9 \mathrm{mmol}, 2.0$ equiv. $)$ was added and the mixture was stirred for $5 \mathrm{~h}$. The residue was evaporated to dryness and directly purified on column chromatography (hexane/EtOAc $=40 / 1$ to $10 / 1$ ). Compound 28 was obtained as a colorless solid (173.2 $\mathrm{mg}$, $34 \%)$. 

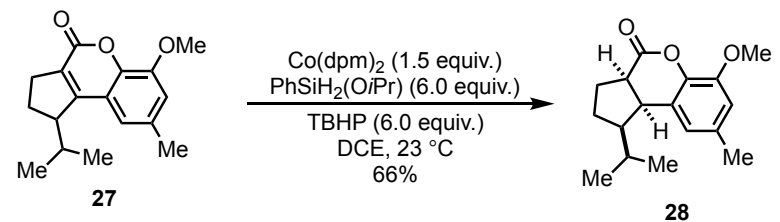

For the $\mathrm{Co}(\mathrm{dpm})_{2}$ procedure: To compound $27(0.50 \mathrm{~g}, 1.8 \mathrm{mmol}, 1.0$ equiv. $)$ in a flame-dried flask was added DCE $(40 \mathrm{~mL})$ in the glovebox. Then to the mixture were added $\operatorname{Co}(\mathrm{dpm})_{2}(234.0$ mg, $0.55 \mathrm{mmol}, 0.3$ equiv.), $\mathrm{PhSiH}_{2}(\mathrm{O} i \mathrm{Pr})(0.40 \mathrm{~mL}, 2.2 \mathrm{mmol}, 1.2$ equiv.) and $t \mathrm{BuOOH}$ (TBHP, $0.40 \mathrm{~mL}, 5.5 \mathrm{M}$ in nonane, $2.2 \mathrm{mmol}, 1.2$ equiv.). The reaction was stirred at room temperature for $1 \mathrm{~h}$. Then to the mixture DCE $(8 \mathrm{~mL}), \mathrm{Co}(\mathrm{dpm})_{2}\left(234.0 \mathrm{mg}, 0.3\right.$ equiv.), $\mathrm{PhSiH}_{2}(\mathrm{O} i \mathrm{Pr})(0.40 \mathrm{~mL}$, 1.2 equiv.) and $t \mathrm{BuOOH}(0.40 \mathrm{~mL}, 5.5 \mathrm{M}$ in nonane, 1.2 equiv. $)$ were added and the reaction was further stirred for $1 \mathrm{~h}$. The addition was repeated for 3 more times until 1.5 equiv. of $\operatorname{Co}(\mathrm{dpm})_{2}$ was added in total. The stirring was continued for $1 \mathrm{~h}$. Then the reaction was quenched with aqueous $\mathrm{HCl}(30 \mathrm{~mL}, 1 \mathrm{M})$ and extracted with $\mathrm{DCM}(30 \mathrm{~mL} \times 3)$. The combined organic phase was dried $\left(\mathrm{Na}_{2} \mathrm{SO}_{4}\right)$, filtered and concentrated. The residue was purified on column chromatography (hexane/EtOAc $=95 / 5$ ). Compound $\mathbf{2 8}$ was obtained as a solid with trace green impurity (322.5 mg, 66\%). ${ }^{1}$ H NMR (500 MHz, CDCl $) \delta 6.64$ (s, 2H), 3.86 (s, 3H), 3.44 (dd, $J=9.0,9.0 \mathrm{~Hz}, 1 \mathrm{H}), 3.04(\mathrm{ddd}, J=9.0,9.0,3.7 \mathrm{~Hz}, 1 \mathrm{H}), 2.45-2.36(\mathrm{~m}, 1 \mathrm{H}), 2.32(\mathrm{~s}, 3 \mathrm{H})$, $2.19-2.10(\mathrm{~m}, 1 \mathrm{H}), 2.06-1.96(\mathrm{~m}, 1 \mathrm{H}), 1.80-1.71(\mathrm{~m}, 1 \mathrm{H}), 1.71-1.60(\mathrm{~m}, 1 \mathrm{H}), 1.55-$ $1.46(\mathrm{~m}, 1 \mathrm{H}), 0.69(\mathrm{~d}, J=6.6 \mathrm{~Hz}, 3 \mathrm{H}), 0.64(\mathrm{~d}, J=6.6 \mathrm{~Hz}, 3 \mathrm{H}) ;{ }^{13} \mathbf{C}$ NMR $\left(\mathbf{1 2 5} \mathbf{~ M H z}, \mathbf{C D C l}_{3}\right)$ $\delta 170.7,147.1,139.0,133.5,123.2,121.5,111.5,55.9,50.5,43.8,42.1,27.9,27.6,27.0,23.4$, 21.3, 19.8. IR (film): 2955, 1751, 1490, 1464, 1359, 1212, 1136, 1039, 1016, 834, 736, 700 $\mathrm{cm}^{-1}$; HRMS (ESI): $\mathrm{m} / z$ Calc. for $\mathrm{C}_{17} \mathrm{H}_{23} \mathrm{O}_{3}[\mathrm{M}+\mathrm{H}]^{+}:$275.1642, found: 275.1643.

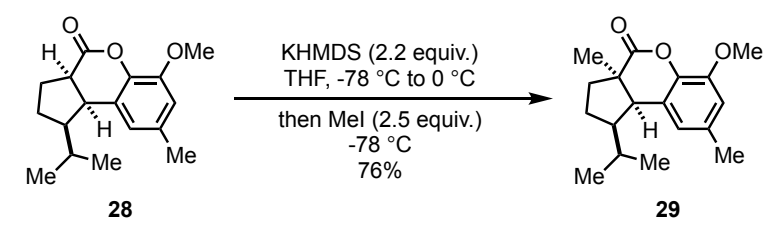

To 28 (20.0 mg, $0.073 \mathrm{mmol}, 1.0$ equiv.) in THF ( $1 \mathrm{~mL}$ ) was added KHMDS (0.16 mL, $1 \mathrm{M}$ in THF, $0.16 \mathrm{mmol}, 2.2$ equiv.) at $-78{ }^{\circ} \mathrm{C}$ under Ar. The reaction was warmed up to $0{ }^{\circ} \mathrm{C}$ and stirred for $40 \mathrm{~min}$. The mixture was cooled down to $-78^{\circ} \mathrm{C}$ and $\mathrm{MeI}(11 \mu \mathrm{L}, 0.18 \mathrm{mmol}, 2.5$ equiv.) was added to the solution. The mixture was stirred at $-78^{\circ} \mathrm{C}$ for $90 \mathrm{~min}$ before it was quenched with saturated aqueous ammonium chloride $(1 \mathrm{~mL})$ and extracted with ether $(2 \mathrm{~mL}$ 
$\times 3)$. The combined organic phase was washed with brine, dried $\left(\mathrm{Na}_{2} \mathrm{SO}_{4}\right)$, filtered and concentrated. The residue was purified on column chromatography (hexane/EtOAc $=25 / 1$ ) and compound 29 was obtained as a white solid (15.9 mg, 76\%). ${ }^{1} \mathbf{H}$ NMR (500 MHz, $\mathbf{C D C l}_{3}$ ) ${ }^{1} \mathrm{H}$ NMR $\left(500 \mathrm{MHz}, \mathrm{CDCl}_{3}\right) \delta 6.64(\mathrm{~s}, 1 \mathrm{H}), 6.58(\mathrm{~s}, 1 \mathrm{H}), 3.86$ (s, 3H), 3.02 (d, $J=10.6 \mathrm{~Hz}$, 1H), $2.69-2.60(\mathrm{~m}, 1 \mathrm{H}), 2.32(\mathrm{~s}, 3 \mathrm{H}), 2.32-2.21(\mathrm{~m}, 1 \mathrm{H}), 1.87-1.74(\mathrm{~m}, 1 \mathrm{H}), 1.64-1.49$ $(\mathrm{m}, 2 \mathrm{H}), 1.45-1.36(\mathrm{~m}, 1 \mathrm{H}), 1.26(\mathrm{~s}, 3 \mathrm{H}), 0.62(\mathrm{~d}, J=6.6 \mathrm{~Hz}, 3 \mathrm{H}), 0.56(\mathrm{~d}, J=6.7 \mathrm{~Hz}, 3 \mathrm{H})$; ${ }^{13}$ C NMR (125 MHz, $\left.\mathbf{C D C l}_{3}\right) \delta 173.4,146.8,138.4,133.5,123.3,122.6,111.5,55.9,51.5$, 49.7, 48.2, 36.9, 28.3, 26.7, 24.1, 23.4, 21.3, 19.1; IR (film): 2958, 2872, 1754, 1602, 1492, 1465, 1350, 1293, 1216, $1135 \mathrm{~cm}^{-1}$; HRMS (ESI): $\mathrm{m} / z$ Calc. for $\mathrm{C}_{18} \mathrm{H}_{25} \mathrm{O}_{3}[\mathrm{M}+\mathrm{H}]^{+}: 289.1798$, found: 289.1799 .

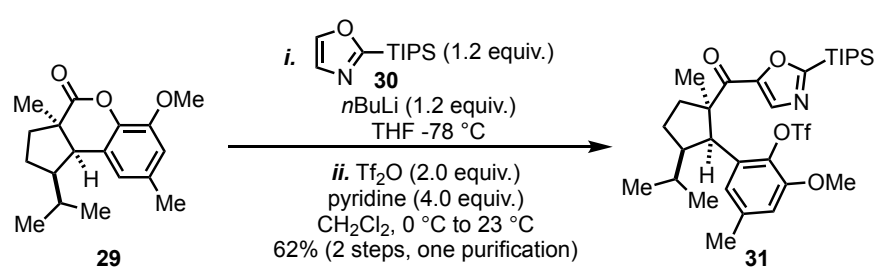

To a flame-dried vial flask were added 2-triisopropylsilyloxazole (30, $25.0 \mathrm{mg}, 0.11 \mathrm{mmol}$, 1.2 equiv.) and THF $(1 \mathrm{~mL})$. The mixture was cooled down to $-30^{\circ}$ and $n \operatorname{BuLi}(0.043 \mathrm{~mL}, 2.6$ $\mathrm{M}$ in hexane, $0.11 \mathrm{mmol}, 1.2$ equiv.) was added dropwise. The resulting mixture was stirred for $30 \mathrm{~min}$, then cooled down to $-78^{\circ} \mathrm{C}$. In another flask compound $29(26.8 \mathrm{mg}, 0.093 \mathrm{mmol}$, 1 equiv.) and THF ( $1 \mathrm{~mL})$ were charged. The mixture was cooled down to $-78{ }^{\circ} \mathrm{C}$ and the lithiated oxazole solution was transferred to the THF solution of $\mathbf{2 9}$ dropwise. The resulting mixture was stirred at this temperature for $1 \mathrm{~h}$ before it was quenched with saturated aqueous ammonium chloride $(1 \mathrm{ml})$ and extracted with ether $(2 \mathrm{~mL} \times 3)$. The combined organic phase was washed with brine $(2 \mathrm{~mL})$, dried $\left(\mathrm{Na}_{2} \mathrm{SO}_{4}\right)$, filtered and concentrated. The crude product was further dried under vacuum to remove the solvent and was then used without further purification.

The above crude residue was dissolved in DCM $(3 \mathrm{~mL})$ and cooled down to $0{ }^{\circ} \mathrm{C}$. Pyridine (30 $\mu \mathrm{L}, 0.37 \mathrm{mmol}, 4.0$ equiv.) and triflic anhydride (30 $\mu \mathrm{L}, 0.18 \mathrm{mmol}, 2.0$ equiv.) were added sequentially. The reaction mixture was warmed up to room temperature and stirred for $7.5 \mathrm{~h}$ before it was quenched with saturated aqueous sodium bicarbonate $(2 \mathrm{~mL})$ and extracted 
with ether $(2 \mathrm{~mL} \times 3)$ The combined organic phase was washed with brine $(2 \mathrm{~mL})$, dried $\left(\mathrm{Na}_{2} \mathrm{SO}_{4}\right)$, filtered and concentrated. The residue was purified on column chromatography (hexane/ether $=7 / 1$ to $5 / 1$ ). Compound 31 was obtained as a white solid $(37.0 \mathrm{mg}, 62 \%) .{ }^{1} \mathbf{H}$ NMR (500 MHz, CDCl $) \delta 7.75(\mathrm{~s}, 1 \mathrm{H}), 6.59$ (s, 1H), $6.36(\mathrm{~s}, 1 \mathrm{H}), 3.81(\mathrm{~s}, 3 \mathrm{H}), 3.70$ (d, $J=$ $6.6 \mathrm{~Hz}, 1 \mathrm{H}), 2.71-2.57(\mathrm{~m}, 1 \mathrm{H}), 2.25(\mathrm{~s}, 3 \mathrm{H}), 2.23-2.06(\mathrm{~m}, 2 \mathrm{H}), 1.85-1.74(\mathrm{~m}, 1 \mathrm{H}), 1.62$ (s, 3H), $1.50-1.37(\mathrm{~m}, 3 \mathrm{H}), 1.17-1.03(\mathrm{~m}, 19 \mathrm{H}), 0.82(\mathrm{~d}, J=6.6 \mathrm{~Hz}, 3 \mathrm{H}), 0.77(\mathrm{~d}, J=6.4$ $\mathrm{Hz}, 3 \mathrm{H}) ;{ }^{13} \mathbf{C}$ NMR (125 MHz, $\left.\mathbf{C D C l}_{3}\right) \delta 191.3,171.5,151.0,150.3,137.7,135.8,135.2$, $133.8,120.9,118.8(\mathrm{q}, J=325 \mathrm{~Hz}), 111.3,60.6,55.7,50.3,49.2,33.7,29.3,28.7,28.43,22.1$, 22.0, 18.3, 10.9; ${ }^{19}$ F NMR (471 MHz, CDCl $) \delta$-73.84; IR (film): 2946, 2869, 1674, 1595, 1545, 1464, 1411, 1326, 1203, 1134, 1110, 878, $627 \mathrm{~cm}^{-1}$; HRMS (ESI): $\mathrm{m} / z$ Calc. for C31H47F3NO6SSi[M+H] $]^{+}$646.2840, found:646.2838.

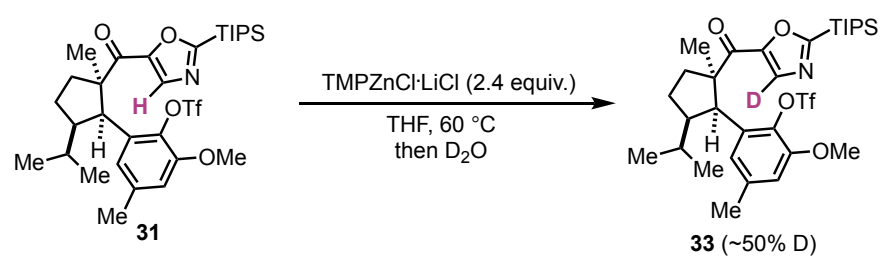

To a flame-dried vial were added compound 31 (3.0 mg, $0.047 \mathrm{mmol}, 1.0$ equiv.) and THF $(0.1 \mathrm{~mL})$. Then TMPZnCl. $\mathrm{LiCl}(20 \mu \mathrm{L}, 0.57 \mathrm{M}$ in THF, $0.11 \mathrm{mmol}, 2.4$ equiv. $)$ was added. The reaction was heated to $60{ }^{\circ} \mathrm{C}$ and stirred for $10 \mathrm{~h}$ before it was cooled down to room temperature, quenched with $\mathrm{D}_{2} \mathrm{O}(0.5 \mathrm{~mL})$, and extracted with ether $(1 \mathrm{~mL} \times 3)$. The combined organic phase was washed with brine, dried $\left(\mathrm{Na}_{2} \mathrm{SO}_{4}\right)$, filtered and concentrated. The deuteration yield was determined as $\sim 50 \%$ by crude ${ }^{1} \mathrm{H}$ NMR.

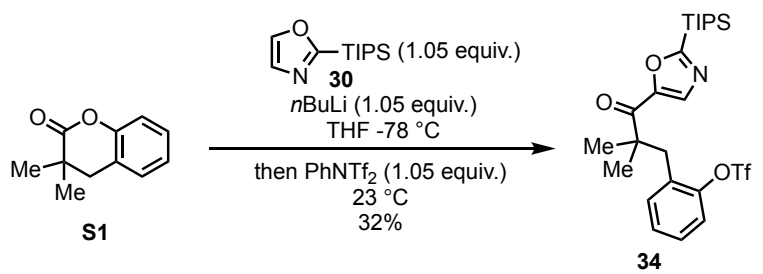

To a flame-dried vial flask were added 2-triisopropylsilyloxazole (30, $5.4 \mathrm{~g}, 23.8 \mathrm{mmol}, 1.05$ equiv.) and THF (100 mL). The mixture was cooled down to $-30{ }^{\circ} \mathrm{C}$ and $n \mathrm{BuLi}(9.5 \mathrm{~mL}, 2.5$ $\mathrm{M}$ in hexane, $23.8 \mathrm{mmol}, 1.05$ equiv.) was added dropwise. The resulting mixture was stirred at this temperature for $60 \mathrm{~min}$ and then cooled down to $-78{ }^{\circ} \mathrm{C}$. In another flask 3,3-dimethyl 
dihydrocoumarin (S1, 4.0 g, 22.7 mmol, 1.0 equiv.) and THF (15 mL) were added. The mixture was cooled down to $-78{ }^{\circ} \mathrm{C}$ and the lithiated oxazole solution was transferred to the THF solution of $\mathbf{S 1}$ dropwise. The mixture was stirred at this temperature for $70 \mathrm{~min}$ and then $\mathrm{PhNTf}_{2}(8.5 \mathrm{~g}, 23.8 \mathrm{mmol}, 1.05$ equiv.) was added. The reaction was warmed up to room temperature and further stirred for $7.5 \mathrm{~h}$ before it was quenched with saturated aqueous ammonium chloride $(200 \mathrm{~mL})$ and extracted with ether $(80 \mathrm{~mL} \times 3)$. The combined organic phase was washed with brine $(80 \mathrm{~mL})$, dried $\left(\mathrm{Na}_{2} \mathrm{SO}_{4}\right)$, filtered and concentrated. The residue was purified on column chromatography (first column: DCM/hexane/ether $=50 / 50 / 1$; second column hexane/EtOAc $=30 / 1)$. Compound 34 was obtained as a light-yellow oil $(5.25 \mathrm{~g}$, 32\%). ${ }^{1} \mathbf{H}$ NMR (500 MHz, $\left.\mathbf{C D C l}_{3}\right) \delta 8.02(\mathrm{~d}, J=2.1 \mathrm{~Hz}, 1 \mathrm{H}), 7.84(\mathrm{~d}, J=2.1 \mathrm{~Hz}, 1 \mathrm{H}), 7.34$ - 7.23 (m, 3H), 7.15 (d, $J=7.8 \mathrm{~Hz}, 1 \mathrm{H}), 3.23$ (s, 2H), 1.32 (s, 6H); ${ }^{13}$ C NMR (125 MHz, $\left.\mathbf{C D C l}_{3}\right) \delta 192.8,152.5,149.2,148.7,134.1,133.0,130.1,128.9,128.1,121.4,118.5(\mathrm{q}, J=$ $319 \mathrm{~Hz}), 48.2,38.2,24.0 ;{ }^{19}$ F NMR (471 MHz, CDCl$) \delta$ ) -74.86; IR (film): 2947, 2869, 1573, 1544, 1421, 1214, 1140, 903, $883 \mathrm{~cm}^{-1}$; HRMS (ESI): $\mathrm{m} / z$ Calc. for $\mathrm{C}_{24} \mathrm{H}_{35} \mathrm{~F}_{3} \mathrm{NO}_{5} \mathrm{SSi}$ $[\mathrm{M}+\mathrm{H}]^{+}:$534.1951, found: 534.1952.

The following $\mathrm{C}-\mathrm{H}$ arylation conditions were explored, but no desired product was formed.

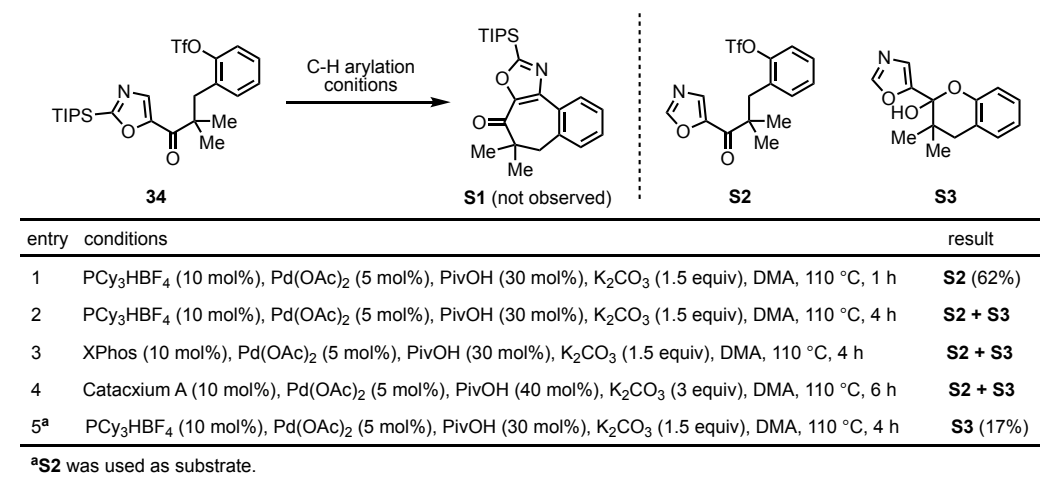

Table S1. Attempted direct C-H arylations 


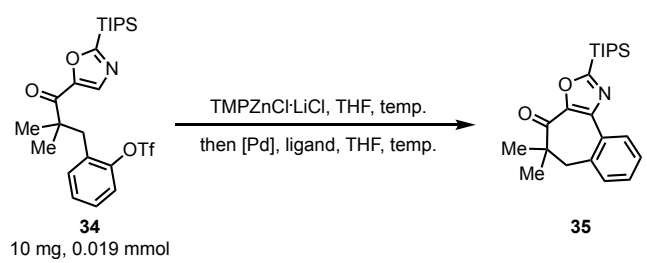

\begin{tabular}{|c|c|c|c|}
\hline entry & TMPZnCI LiCl, conc.,temp, time & Pd cat., ligand, conc., temp, time & JMR yield of 35 \\
\hline 1 & 2.0 equiv., $0.04 \mathrm{M}, 60^{\circ} \mathrm{C}, 21 \mathrm{~h}$ & 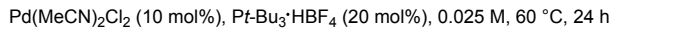 & $0 \%$ \\
\hline 2 & 2.0 equiv., $0.04 \mathrm{M}, 60^{\circ} \mathrm{C}, 21 \mathrm{~h}$ & $\mathrm{Pd}(\mathrm{MeCN})_{2} \mathrm{Cl}_{2}(10 \mathrm{~mol} \%), \mathrm{Cy}_{2} \mathrm{P}\left(\mathrm{CH}_{2}\right)_{3} \mathrm{PCy}_{2}(20 \mathrm{~mol} \%), 0.025 \mathrm{M}, 60^{\circ} \mathrm{C}, 24 \mathrm{~h}$ & $0 \%$ \\
\hline 3 & 2.0 equiv., $0.04 \mathrm{M}, 60^{\circ} \mathrm{C}, 21 \mathrm{~h}$ & $\mathrm{Pd}(\mathrm{MeCN})_{2} \mathrm{Cl}_{2}(10 \mathrm{~mol} \%)$, SPhos $(20 \mathrm{~mol} \%), 0.025 \mathrm{M}, 60^{\circ} \mathrm{C}, 24 \mathrm{~h}$ & $19 \%$ \\
\hline 4 & 2.0 equiv., $0.04 \mathrm{M}, 60^{\circ} \mathrm{C}, 21 \mathrm{~h}$ & $\mathrm{Pd}(\mathrm{MeCN})_{2} \mathrm{Cl}_{2}(10 \mathrm{~mol} \%)$, RuPhos $(20 \mathrm{~mol} \%), 0.025 \mathrm{M}, 60^{\circ} \mathrm{C}, 24 \mathrm{~h}$ & $8 \%$ \\
\hline 5 & 2.0 equiv., $0.04 \mathrm{M}, 60^{\circ} \mathrm{C}, 21 \mathrm{~h}$ & $\mathrm{Pd}(\mathrm{MeCN})_{2} \mathrm{Cl}_{2}(10 \mathrm{~mol} \%)$, JohnPhos (20 mol\%), $0.025 \mathrm{M}, 60^{\circ} \mathrm{C}, 24 \mathrm{~h}$ & $11 \%$ \\
\hline 6 & 2.0 equiv., $0.04 \mathrm{M}, 60^{\circ} \mathrm{C}, 21 \mathrm{~h}$ & $\mathrm{Pd}(\mathrm{MeCN})_{2} \mathrm{Cl}_{2}(10 \mathrm{~mol} \%)$, tBuBrettPhos $(20 \mathrm{~mol} \%), 0.025 \mathrm{M}, 60{ }^{\circ} \mathrm{C}, 24 \mathrm{~h}$ & $8 \%$ \\
\hline 7 & 2.0 equiv., $0.04 \mathrm{M}, 60^{\circ} \mathrm{C}, 21 \mathrm{~h}$ & $\mathrm{Pd}(\mathrm{MeCN})_{2} \mathrm{Cl}_{2}(10 \mathrm{~mol} \%)$, XantPhos $(20 \mathrm{~mol} \%), 0.025 \mathrm{M}, 60^{\circ} \mathrm{C}, 24 \mathrm{~h}$ & $0 \%$ \\
\hline 8 & 2.0 equiv., $0.04 \mathrm{M}, 60^{\circ} \mathrm{C}, 21 \mathrm{~h}$ & $\mathrm{Pd}(\mathrm{MeCN})_{2} \mathrm{Cl}_{2}(10 \mathrm{~mol} \%), \mathrm{XPhos}(20 \mathrm{~mol} \%), 0.025 \mathrm{M}, 60^{\circ} \mathrm{C}, 24 \mathrm{~h}$ & $13 \%$ \\
\hline 9 & 2.0 equiv., $0.04 \mathrm{M}, 60^{\circ} \mathrm{C}, 21 \mathrm{~h}$ & XPhos Pd G2 (10 mol\%), XPhos (10 mol\%), $0.025 \mathrm{M}, 60^{\circ} \mathrm{C}, 24 \mathrm{~h}$ & $46 \%$ \\
\hline 10 & 2.0 equiv., $0.04 \mathrm{M}, 60^{\circ} \mathrm{C}, 21 \mathrm{~h}$ & $\mathrm{Pd}(\mathrm{dtbpf}) \mathrm{Cl}_{2}(10 \mathrm{~mol} \%), 0.025 \mathrm{M}, 60^{\circ} \mathrm{C}, 24 \mathrm{~h}$ & $<5 \%$ \\
\hline 11 & 2.0 equiv., $0.04 \mathrm{M}, 60^{\circ} \mathrm{C}, 21 \mathrm{~h}$ & PEPPSI-IPr (10 mol\%), $0.025 \mathrm{M}, 60^{\circ} \mathrm{C}, 24 \mathrm{~h}$ & $0 \%$ \\
\hline 12 & 2.0 equiv., $0.04 \mathrm{M}, 60^{\circ} \mathrm{C}, 21 \mathrm{~h}$ & Pd-PEPPSI-IPent (10 mol\%), $0.025 \mathrm{M}, 60^{\circ} \mathrm{C}, 24 \mathrm{~h}$ & $0 \%$ \\
\hline 13 & 2.0 equiv., $0.04 \mathrm{M}, 60^{\circ} \mathrm{C}, 22 \mathrm{~h}$ & $\mathrm{Pd}(\mathrm{MeCN})_{2} \mathrm{Cl}_{2}(10 \mathrm{~mol} \%), \mathrm{XPhos}(20 \mathrm{~mol} \%), 0.025 \mathrm{M}, 60^{\circ} \mathrm{C}, 22 \mathrm{~h}$ & $16 \%$ \\
\hline 14 & 2.0 equiv., $0.04 \mathrm{M}, 60^{\circ} \mathrm{C}, 22 \mathrm{~h}$ & $\mathrm{Pd}_{2}(\mathrm{dba})_{3}(10 \mathrm{~mol} \%), \mathrm{XPhos}(20 \mathrm{~mol} \%), 0.025 \mathrm{M}, 60^{\circ} \mathrm{C}, 22 \mathrm{~h}$ & $9 \%$ \\
\hline 15 & 2.0 equiv., $0.04 \mathrm{M}, 60^{\circ} \mathrm{C}, 22 \mathrm{~h}$ & $\mathrm{Pd}(\mathrm{dba})_{2}(10 \mathrm{~mol} \%), \mathrm{XPhos}(20 \mathrm{~mol} \%), 0.025 \mathrm{M}, 60{ }^{\circ} \mathrm{C}, 22 \mathrm{~h}$ & $9 \%$ \\
\hline 16 & 2.0 equiv., $0.04 \mathrm{M}, 60^{\circ} \mathrm{C}, 22 \mathrm{~h}$ & $\mathrm{Pd}(\mathrm{OAc})_{2}(10 \mathrm{~mol} \%), \mathrm{XPhos}(20 \mathrm{~mol} \%), 0.025 \mathrm{M}, 60^{\circ} \mathrm{C}, 22 \mathrm{~h}$ & $34 \%$ \\
\hline 17 & 2.0 equiv., $0.04 \mathrm{M}, 60{ }^{\circ} \mathrm{C}, 22 \mathrm{~h}$ & $\mathrm{Pd}(\mathrm{TFA})_{2}(10 \mathrm{~mol} \%), \mathrm{XPhos}(20 \mathrm{~mol} \%), 0.025 \mathrm{M}, 60^{\circ} \mathrm{C}, 22 \mathrm{~h}$ & $25 \%$ \\
\hline 18 & 2.0 equiv., $0.04 \mathrm{M}, 60^{\circ} \mathrm{C}, 22 \mathrm{~h}$ & XPhos Pd G2 (10 mol\%), XPhos (10 mol\%), $0.025 \mathrm{M}, 60^{\circ} \mathrm{C}, 22 \mathrm{~h}$ & $55 \%$ \\
\hline 19 & 2.0 equiv., $0.04 \mathrm{M}, 60^{\circ} \mathrm{C}, 12 \mathrm{~h}$ & XPhos Pd G2 (10 mol\%), XPhos (10 mol\%), $0.025 \mathrm{M}, 40^{\circ} \mathrm{C}, 7.5 \mathrm{~h}$ & $52 \%$ \\
\hline 20 & 2.0 equiv., $0.04 \mathrm{M}, 60^{\circ} \mathrm{C}, 12 \mathrm{~h}$ & XPhos Pd G2 (10 mol\%), XPhos (10 mol\%), $0.025 \mathrm{M}, 50^{\circ} \mathrm{C}, 7.5 \mathrm{~h}$ & $51 \%$ \\
\hline 21 & 2.0 equiv., $0.04 \mathrm{M}, 60^{\circ} \mathrm{C}, 12 \mathrm{~h}$ & XPhos Pd G2 (10 mol\%), XPhos (10 mol\%), $0.025 \mathrm{M}, 60^{\circ} \mathrm{C}, 7.5 \mathrm{~h}$ & $56 \%$ \\
\hline 22 & 2.0 equiv., $0.04 \mathrm{M}, 60^{\circ} \mathrm{C}, 12 \mathrm{~h}$ & XPhos Pd G2 (10 mol\%), XPhos (10 mol\%), $0.025 \mathrm{M}, 70^{\circ} \mathrm{C}, 7.5 \mathrm{~h}$ & $59 \%$ \\
\hline 23 & 2.0 equiv., $0.04 \mathrm{M}, 60^{\circ} \mathrm{C}, 12 \mathrm{~h}$ & XPhos Pd G3 (10 mol\%), XPhos (10 mol\%), $0.025 \mathrm{M}, 60^{\circ} \mathrm{C}, 7.5 \mathrm{~h}$ & $46 \%$ \\
\hline 24 & 2.0 equiv., $0.12 \mathrm{M}, 60^{\circ} \mathrm{C}, 12 \mathrm{~h}$ & XPhos Pd G2 (10 mol\%), XPhos (10 mol\%), (0.078 M), $60{ }^{\circ} \mathrm{C}, 7.5 \mathrm{~h}$ & $<5 \%$ \\
\hline 25 & 2.0 equiv., $0.04 \mathrm{M}, 60^{\circ} \mathrm{C}, 14 \mathrm{~h}$ & XPhos Pd G2 (10 mol\%), XPhos (10 mol\%), $0.025 \mathrm{M}, 60^{\circ} \mathrm{C}, 12.5 \mathrm{~h}$ & $58 \%$ \\
\hline 26 & 2.0 equiv., $0.04 \mathrm{M}, 60^{\circ} \mathrm{C}, 14 \mathrm{~h}$ & XPhos Pd G2 (10 mol\%), XPhos (10 mol\%) THF/DMI $(2: 1,0.025 \mathrm{M}), 60^{\circ} \mathrm{C}, 12.5 \mathrm{~h}$ & $30 \%$ \\
\hline 27 & 3.4 equiv., $0.09 \mathrm{M}, 50^{\circ} \mathrm{C}, 20 \mathrm{~h}$ & XPhos Pd G2 (10 mol\%), XPhos (10 mol\%), (0.06 M), $50^{\circ} \mathrm{C}, 10 \mathrm{~h}$ & $93 \%$ \\
\hline 28 & 2.4 equiv., $0.09 \mathrm{M}, 50{ }^{\circ} \mathrm{C}, 20 \mathrm{~h}$ & XPhos Pd G2 (10 mol\%), XPhos (10 mol\%), (0.06 M), $50{ }^{\circ} \mathrm{C}, 10 \mathrm{~h}$ & $85 \%$ \\
\hline 29 & 1.4 equiv., $0.09 \mathrm{M}, 50^{\circ} \mathrm{C}, 20 \mathrm{~h}$ & XPhos Pd G2 (10 mol\%), XPhos (10 mol\%), (0.06 M), $50^{\circ} \mathrm{C}, 10 \mathrm{~h}$ & $52 \%$ \\
\hline 30 & 1.4 equiv., $0.09 \mathrm{M}, 75^{\circ} \mathrm{C}, 20 \mathrm{~h}$ & XPhos Pd G2 (10 mol\%), XPhos (10 mol\%), (0.06 M), $50^{\circ} \mathrm{C}, 10 \mathrm{~h}$ & $75 \%$ \\
\hline
\end{tabular}<smiles></smiles>

JohnPhos

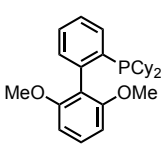

SPhos

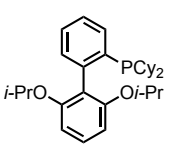

RuPhos

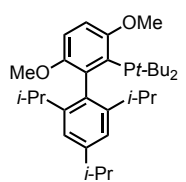

tBuBrettPhos

Table S2. Reaction condition optimization of the C-H metalation/Negishi cross coupling

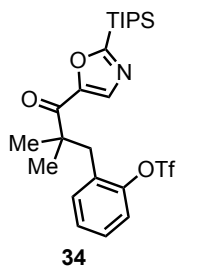

34

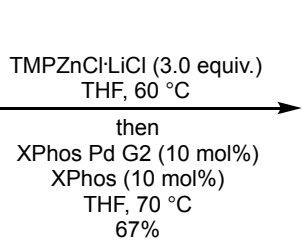

$67 \%$
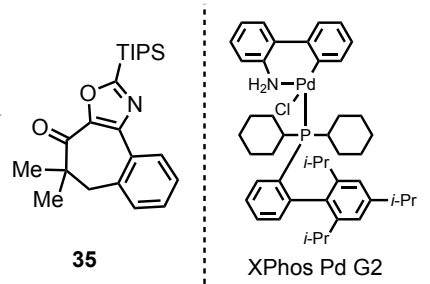

To a flame-dried vial were added compound 34 (0.92 g, 1.72 mmol, 1.0 equiv.) and THF (10 $\mathrm{mL})$. Then TMPZnCl$\cdot \mathrm{LiCl}(6.2 \mathrm{~mL}, 0.84 \mathrm{M}$ in THF, $5.2 \mathrm{mmol}, 3.0$ equiv.) was added. The reaction mixture was warmed up to $60{ }^{\circ} \mathrm{C}$ and stirred for $14 \mathrm{~h}$. Then the residue was cooled down to room temperature and a solution of XPhos Pd G2 (135.6 mg, $0.172 \mathrm{mmol}, 10 \mathrm{~mol} \%)$ 
and XPhos (82.2 mg, $0.172 \mathrm{mmol}, 10 \mathrm{~mol} \%)$ in THF ( $3 \mathrm{~mL}$ ) was added. The reaction mixture was warmed up to $70{ }^{\circ} \mathrm{C}$ and stirred for $11.5 \mathrm{~h}$ before it was cooled down to room temperature, quenched with saturated aqueous ammonium chloride $(10 \mathrm{~mL})$ and extracted with ether $(10$ $\mathrm{mL} \times 3)$. The combined organic phase was washed with brine, dried $\left(\mathrm{Na}_{2} \mathrm{SO}_{4}\right)$, filtered and concentrated. The residue was purified on column chromatography (hexane/ether $=50 / 1$ to 25/1). Compound 35 was obtained as a light-yellow oil (440.3 mg, 67\%). ${ }^{1}$ H NMR (500 MHz, $\left.\mathbf{C D C l}_{3}\right) \delta 8.23(\mathrm{dd}, J=7.6,1.5 \mathrm{~Hz}, 1 \mathrm{H}), 7.42(\mathrm{ddd}, J=7.6,7.5,1.4 \mathrm{~Hz}, 1 \mathrm{H}), 7.34(\mathrm{ddd}$, $J=7.4,7.4,1.5 \mathrm{~Hz}, 1 \mathrm{H}), 7.26(\mathrm{dd}, J=7.5,1.5 \mathrm{~Hz}, 1 \mathrm{H}), 3.01(\mathrm{br}, 2 \mathrm{H}), 1.54-1.43(\mathrm{~m}, 3 \mathrm{H})$, 1.20 (d, $J=7.5 \mathrm{~Hz}, 18 \mathrm{H}), 1.28-1.08$ (br, 6H); ${ }^{13} \mathbf{C}$ NMR (125 MHz, CDCl $) \delta$ 192.7, 172.8, 145.1, 143.6, 137.2, 131.0, 129.7, 129.5, 127.9, 127.2, 44.0, 43.7, 18.3, 10.9; IR (film): 2944, 2867, 1666, 1467, 1385, 1360, 1236, 1030, 958, 883, 764, $675 \mathrm{~cm}^{-1}$; HRMS (ESI): $\mathrm{m} / \mathrm{z}$ Calc. for $\mathrm{C}_{23} \mathrm{H}_{34} \mathrm{NO}_{2} \mathrm{Si}[\mathrm{M}+\mathrm{H}]^{+}: 384.2353$, found: 384.2354 .
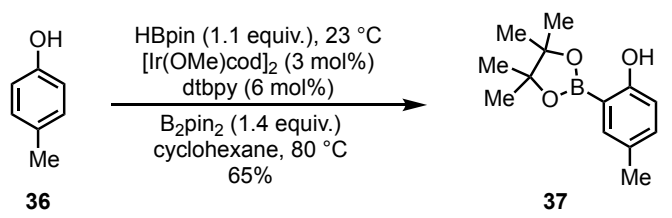

Compound 37 was prepared through modification of the reported method. ${ }^{[5]}$

To $p$-cresol (36, $5.40 \mathrm{~g}, 50 \mathrm{mmol}, 1.0$ equiv.) in a flame-dried seal-tube was added HBpin (8.0 $\mathrm{mL}, 55 \mathrm{mmol}, 1.1$ equiv.) and the mixture was stirred at room temperature overnight. To this mixture, $[\mathrm{Ir}(\mathrm{OMe})(\mathrm{cod})]_{2}(0.99 \mathrm{~g}, 1.5 \mathrm{mmol}, 3.0 \mathrm{~mol} \%)$, dtbpy $(0.80 \mathrm{~g}, 3.0 \mathrm{mmol}, 6.0 \mathrm{~mol} \%)$, and $\mathrm{B}_{2} \operatorname{pin}_{2}(17.80 \mathrm{~g}, 70 \mathrm{mmol}, 1.4$ equiv. $)$ were charged. Dry cyclohexane $(150 \mathrm{~mL})$ was added under an inert atmosphere. The seal-tube was capped and warmed up to $80{ }^{\circ} \mathrm{C}$. The mixture was stirred for $24 \mathrm{~h}$ before it was cooled down to room temperature and concentrated. The residue was purified on column chromatography $\left(\mathrm{CHCl}_{3} /\right.$ hexane $\left.=1 / 2\right)$. Compound 37 was obtained as a colorless oil $(7.56 \mathrm{~g}, 65 \%)$. The characterization data was in accordance with the previous report.

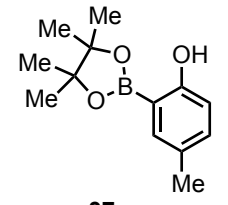

37

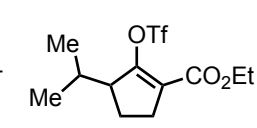

14

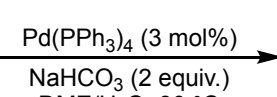
$\mathrm{DME} / \mathrm{H}_{2} \mathrm{O}, 80^{\circ} \mathrm{C}$ $92 \%$

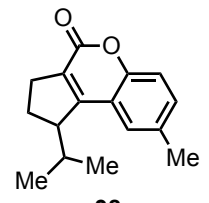

38 
To compound 14 (8.70 g, $26.3 \mathrm{mmol}, 1.0$ equiv.), compound 37 (6.78 g, $29.0 \mathrm{mmol}, 1.1$ equiv.), $\mathrm{Pd}\left(\mathrm{PPh}_{3}\right)_{4}(0.91 \mathrm{~g}, 0.79 \mathrm{mmol}, 3 \mathrm{~mol} \%)$ and sodium bicarbonate $(4.43 \mathrm{~g}, 52.7 \mathrm{mmol}$, 2 equiv.) in the reaction flask were added DME $(125 \mathrm{~mL})$ and water $(25 \mathrm{~mL}$, degassed by bubbling with Ar). The reaction mixture was warmed up to $80{ }^{\circ} \mathrm{C}$ and stirred for $30 \mathrm{~min}$ before it was cooled down to room temperature, diluted with water $(100 \mathrm{~mL})$ and extracted with ether $(60 \mathrm{~mL} \times 3)$. The combined organic phase was washed with brine $(100 \mathrm{~mL})$, dried $\left(\mathrm{Na}_{2} \mathrm{SO}_{4}\right)$, filtered and concentrated. The residue was then dissolved in DCM $(40 \mathrm{~mL})$, filtered to remove the remaining salts and concentrated again. Purification was done through recrystallization with DCM $(\sim 15 \mathrm{~mL})$. The crude residue was dissolved in DCM at reflux, cooled down to room temperature slowly and recrystallized in the $-20{ }^{\circ} \mathrm{C}$ refrigerator overnight. The mother liquid was removed carefully, and the remaining crystal was washed with cold ethyl acetate to obtain compound $\mathbf{3 8}$ as white crystalline solid (4.28 $\mathrm{g}$ ). Then the mother liquid was concentrated and purified on column chromatography (hexane/DCM/EtOAc $=45 / 15 / 2)$ to obtain a second portion of compound $38(1.62 \mathrm{~g})$. Yield (combined): 92\%. mp 143-144 ${ }^{\circ} \mathrm{C} ;{ }^{1} \mathbf{H}$ NMR (500 MHz, $\mathbf{C D C l}_{3}$ ) $\delta 7.30$ (s, 1H), $7.28-7.26$ (m, 2H), $3.54-3.40(\mathrm{~m}, 1 \mathrm{H}), 2.82(\mathrm{dd}, J=10.2,2.1 \mathrm{~Hz}, 2 \mathrm{H}), 2.41(\mathrm{~s}, 3 \mathrm{H}), 2.34(\mathrm{ddd}, J=$ $10.2,6.9,3.5 \mathrm{~Hz}, 1 \mathrm{H}), 2.18-2.07(\mathrm{~m}, 2 \mathrm{H}), 1.12(\mathrm{~d}, J=6.9 \mathrm{~Hz}, 3 \mathrm{H}), 0.72(\mathrm{~d}, J=6.8 \mathrm{~Hz}, 3 \mathrm{H})$; ${ }^{13}$ C NMR (125 MHz, $\left.\mathbf{C D C l}_{3}\right) \delta 160.6,157.6,152.6,133.6,131.6,128.0,124.5,118.1,116.8$, 51.2, 30.7, 30.4, 23.5, 22.0, 21.0, 16.2; IR (film): 2960, 2903, 1717, 1574, 1387, 1196, 1137, 1057, 1040, 1002, $950 \mathrm{~cm}^{-1}$; HRMS (ESI): $m / z$ Calc. for $\mathrm{C}_{16} \mathrm{H}_{19} \mathrm{O}_{2}[\mathrm{M}+\mathrm{H}]^{+}: 243.1379$, found: 243.1378.

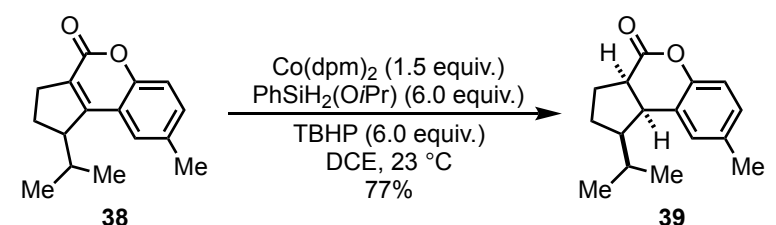

To compound 38 ( $1.00 \mathrm{~g}, 4.1 \mathrm{mmol}, 1.0$ equiv.) in a flame-dried flask was added DCE (15 mL) in the glovebox. Then to the mixture were added $\mathrm{Co}(\mathrm{dpm})_{2}(0.52 \mathrm{~g}, 1.2 \mathrm{mmol}, 0.3$ equiv. $)$, $\mathrm{PhSiH}_{2}(\mathrm{O} i \mathrm{Pr})(0.90 \mathrm{~mL}, 5.0 \mathrm{mmol}, 1.2$ equiv.) and $t \mathrm{BuOOH}$ (TBHP, $0.9 \mathrm{~mL}, 5.5 \mathrm{M}$ in nonane, $5.0 \mathrm{mmol}, 1.2$ equiv.). The reaction was stirred at room temperature for $1 \mathrm{~h}$. Then to the 
mixture DCE (15 mL), Co(dpm $)_{2}(0.52 \mathrm{~g}, 0.3$ equiv. $), \mathrm{PhSiH}_{2}(\mathrm{O} i \mathrm{Pr})(0.9 \mathrm{~mL}, 1.2$ equiv. $)$ and $t \mathrm{BuOOH}(0.9 \mathrm{~mL}, 5.5 \mathrm{M}$ in nonane, 1.2 equiv.) were added and the reaction was further stirred for $1 \mathrm{~h}$. The addition was repeated for 3 more times until 1.5 equiv. of $\mathrm{Co}(\mathrm{dpm})_{2}$ was added in total. The stirring was continued for $1 \mathrm{~h}$. Then the reaction was quenched with aqueous $\mathrm{HCl}(50 \mathrm{~mL}, 1 \mathrm{M})$ and extracted with $\mathrm{DCM}(50 \mathrm{~mL} \times 3)$. The combined organic phase was dried $\left(\mathrm{Na}_{2} \mathrm{SO}_{4}\right)$, filtered and concentrated. The residue was purified on column chromatography (first column: hexane/ether $=15 / 1$; second column: DCM/toluene $=1 / 5$ ). Compound 39 was obtained as a white solid (776.6 mg, 77\%). mp 51-52 ${ }^{\circ} \mathrm{C} ;{ }^{1} \mathbf{H}$ NMR (500 MHz, $\left.\mathbf{C D C l}_{3}\right) \delta 7.05(\mathrm{~s}, 1 \mathrm{H}), 7.03(\mathrm{~d}, J=8.1 \mathrm{~Hz}, 1 \mathrm{H}), 6.89(\mathrm{~d}, J=8.1 \mathrm{~Hz}, 1 \mathrm{H}), 3.46(\mathrm{dd}, J=$ 9.0, $9.0 \mathrm{~Hz}, 1 \mathrm{H}), 3.05(\mathrm{dt}, J=9.0,4.0 \mathrm{~Hz}, 1 \mathrm{H}), 2.40-2.33(\mathrm{~m}, 1 \mathrm{H}), 2.32(\mathrm{~s}, 3 \mathrm{H}), 2.18-2.10$ (m, 1H), $2.07-1.98(\mathrm{~m}, 1 \mathrm{H}), 1.81-1.72(\mathrm{~m}, 1 \mathrm{H}), 1.68-1.58(\mathrm{~m}, 1 \mathrm{H}), 1.52(\mathrm{dq}, J=13.5$, $6.7 \mathrm{~Hz}, 1 \mathrm{H}), 0.71(\mathrm{~d}, J=6.7 \mathrm{~Hz}, 3 \mathrm{H}), 0.63(\mathrm{~d}, J=6.6 \mathrm{~Hz}, 3 \mathrm{H}) ;{ }^{13} \mathbf{C} \mathbf{N M R}\left(\mathbf{1 2 5} \mathbf{M H z}, \mathbf{C D C l}_{\mathbf{3}}\right)$ $\delta 171.5,149.7,133.6,130.2,128.8,122.2,116.6,50.7,43.4,42.4,28.0,27.7,26.9,23.3,20.8$, 19.8; IR (film): 2955, 2871, 1755, 1496, 1212, 1133, 1038, 614, $480 \mathrm{~cm}^{-1}$; HRMS (ESI): $\mathrm{m} / \mathrm{z}$ Calc. for $\mathrm{C}_{16} \mathrm{H}_{21} \mathrm{O}_{2}[\mathrm{M}+\mathrm{H}]^{+}: 245.1536$, found: 245.1537 .
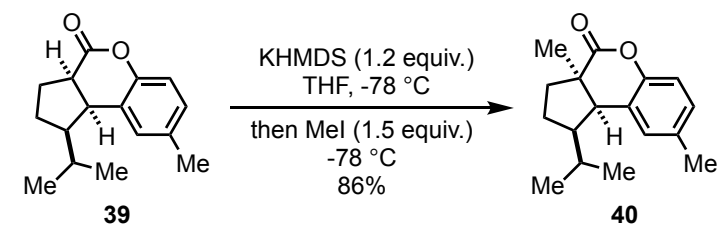

To compound 39 (2.66 g, 10.9 mmol, 1.0 equiv.) in THF (100 mL) was added KHMDS (16.5 $\mathrm{mL}, 1 \mathrm{M}$ in THF, $16.5 \mathrm{mmol}, 1.2$ equiv. $)$ at $-78{ }^{\circ} \mathrm{C}$. After $1 \mathrm{~h}$, MeI $(1.2 \mathrm{~mL}, 19.6 \mathrm{mmol}, 1.5$ equiv.) was added to the solution dropwise. The mixture was stirred at this temperature for 40 min before it was carefully quenched with saturated aqueous ammonium chloride $(40 \mathrm{~mL})$. The mixture was warmed up to room temperature slowly and extracted with ether $(40 \mathrm{~mL} \times 3)$. The combined organic phase was washed with brine $(80 \mathrm{~mL})$, dried $\left(\mathrm{Na}_{2} \mathrm{SO}_{4}\right)$, filtered and concentrated. The residue was purified on column chromatography (hexane/EtOAc $=100 / 0$ to 93/7). Compound 40 was obtained as a white solid (2.43 g, 86\%). mp $64-65{ }^{\circ} \mathrm{C} ;{ }^{1} \mathbf{H}$ NMR $\left(\mathbf{5 0 0} \mathbf{M H z}, \mathbf{C D C l}_{3}\right) \delta 7.03(\mathrm{~d}, J=8.2 \mathrm{~Hz}, 1 \mathrm{H}), 7.00(\mathrm{~s}, 1 \mathrm{H}), 6.90(\mathrm{~d}, J=8.2 \mathrm{~Hz}, 1 \mathrm{H}), 3.03(\mathrm{~d}$, $J=10.7 \mathrm{~Hz}, 1 \mathrm{H}), 2.68-2.62(\mathrm{~m}, 1 \mathrm{H}), 2.32(\mathrm{~s}, 3 \mathrm{H}), 2.27(\mathrm{ddd}, J=10.7,8.2,6.6 \mathrm{~Hz}, 1 \mathrm{H}), 1.83$ 
$-1.75(\mathrm{~m}, 1 \mathrm{H}), 1.63-1.50(\mathrm{~m}, 2 \mathrm{H}), 1.45-1.36(\mathrm{~m}, 1 \mathrm{H}), 1.26(\mathrm{~s}, 3 \mathrm{H}), 0.63(\mathrm{~d}, J=6.6 \mathrm{~Hz}$ 3H), $0.54(\mathrm{~d}, J=6.6 \mathrm{~Hz}, 3 \mathrm{H}) ;{ }^{13} \mathbf{C}$ NMR (125 MHz, $\left.\mathbf{C D C l}_{3}\right) \delta 174.2,149.1,133.5,131.3$, 128.8, 122.3, 116.1, 51.2, 49.7, 48.5, 36.9, 28.2, 26.6, 24.2, 23.4, 20.8, 18.9. IR (film): 2957, 2927, 2871, 1757, 1497, 1214, 1133, $1113 \mathrm{~cm}^{-1}$; HRMS (ESI): $\mathrm{m} / z$ Calc. for $\mathrm{C}_{17} \mathrm{H}_{22} \mathrm{O}_{2}$ $[\mathrm{M}+\mathrm{H}]^{+}:$259.1693, found: 259.1694 .

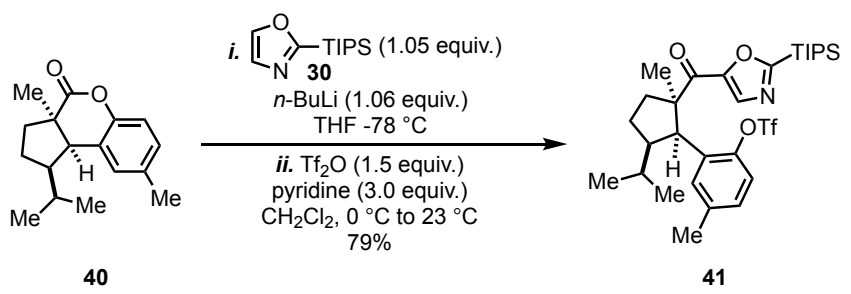

To a flame-dried flask were added 2-triisopropylsilyloxazole (30, $2.23 \mathrm{~g}, 9.88 \mathrm{mmol}, 1.05$ equiv.) and THF $(50 \mathrm{~mL})$. The mixture was cooled down to $-30^{\circ}$ and $n \operatorname{BuLi}(4.0 \mathrm{~mL}, 2.5 \mathrm{M}$ in THF, $10 \mathrm{mmol}, 1.06$ equiv.) was added dropwise. The reaction was stirred at this temperature for $1 \mathrm{~h}$ before it was cooled down to $-78^{\circ} \mathrm{C}$ and carefully diluted with THF (15 $\mathrm{mL})$. To another flask compound 40 (2.43 g, $9.41 \mathrm{mmol}, 1.0$ equiv.) and THF (50 mL) were charged. The mixture was cooled down to $-78{ }^{\circ} \mathrm{C}$ and the lithiated oxazole solution was transferred to the THF solution of compound $\mathbf{4 0}$ dropwise. The reaction mixture was stirred at this temperature for $30 \mathrm{~min}$ before it was quenched with saturated aqueous ammonium chloride $(50 \mathrm{~mL})$ and extracted with ether $(80 \mathrm{~mL} \times 3)$. The combined organic phase was washed with brine $(50 \mathrm{~mL} \times 3)$, dried $\left(\mathrm{Na}_{2} \mathrm{SO}_{4}\right)$, filtered and concentrated. The residue was azeotroped with toluene for 5 times and was then dried under vacuum overnight. The crude residue was directly used in the next step without further purification.

The above crude residue was dissolved in DCM $(120 \mathrm{~mL})$ and cooled down to $0{ }^{\circ} \mathrm{C}$. Pyridine (2.3 mL, $28 \mathrm{mmol}, 3.0$ equiv.) and triflic anhydride ( $2.4 \mathrm{~mL}, 14 \mathrm{mmol}, 1.5$ equiv.) were added dropwise. The mixture was slowly warmed up to room temperature and stirred for $14 \mathrm{~h}$ before it was quenched with saturated aqueous sodium bicarbonate $(50 \mathrm{~mL})$ and extracted with DCM $(60 \mathrm{~mL} \times 3)$. The combined organic phase was washed with brine $(30 \mathrm{~mL})$, dried $\left(\mathrm{Na}_{2} \mathrm{SO}_{4}\right)$, filtered and concentrated. The residue was purified on column chromatography (hexane/ ether $=30 / 1$ to 15/1). Compound 41 was obtained as a light-yellow solid (4.60 g, 79\%). mp 76-77 ${ }^{\circ} \mathrm{C} ;{ }^{1} \mathbf{H}$ NMR (500 MHz, $\left.\mathbf{C D C l}_{3}\right) \delta 7.74(\mathrm{~s}, 1 \mathrm{H}), 7.02(\mathrm{~d}, J=8.4 \mathrm{~Hz}, 1 \mathrm{H}), 6.96(\mathrm{dd}, J=8.4$, 
$2.7 \mathrm{~Hz}, 1 \mathrm{H}), 6.81(\mathrm{~d}, J=2.7 \mathrm{~Hz}, 1 \mathrm{H}), 3.73(\mathrm{~d}, J=6.7 \mathrm{~Hz}, 1 \mathrm{H}), 2.78-2.65(\mathrm{~m}, 1 \mathrm{H}), 2.26(\mathrm{~s}$, $3 \mathrm{H}), 2.36-2.16(\mathrm{~m}, 2 \mathrm{H}), 2.07(\mathrm{ddd}, J=14.4,9.3,5.4 \mathrm{~Hz}, 1 \mathrm{H}), 1.86-1.74(\mathrm{~m}, 1 \mathrm{H}), 1.63(\mathrm{~s}$, 3H), $1.46-1.34(\mathrm{~m}, 3 \mathrm{H}), 1.18-1.07(\mathrm{~m}, 18 \mathrm{H}), 0.84(\mathrm{~d}, J=6.5 \mathrm{~Hz}, 3 \mathrm{H}), 0.76(\mathrm{~d}, J=6.4 \mathrm{~Hz}$, 3H); ${ }^{13} \mathbf{C}$ NMR (125 MHz, $\left.\mathbf{C D C l}_{3}\right) \delta$ 191.3, 171.6, 150.7, 146.7, 137.3, 133.6, 133.3, 130.1, $128.2,120.2,118.4(\mathrm{q}, J=325 \mathrm{~Hz}), 60.7,50.3,49.1,33.3,29.4,28.7,28.4,21.9,21.9,21.4$, 18.3, 10.9; ${ }^{19}$ F NMR (471 MHz, $\mathbf{C D C l}_{3}$ ) $\delta=-76.21$; IR (film): 2947, 2869, 1674, 1416, 1208, 1142, 882, $624 \mathrm{~cm}^{-1}$; HRMS (ESI): $\mathrm{m} / z$ Calc. for $\mathrm{C}_{30} \mathrm{H}_{45} \mathrm{~F}_{3} \mathrm{NO}_{5} \mathrm{SSi}[\mathrm{M}+\mathrm{H}]^{+}: 616.2734$, found: 616.2732 .

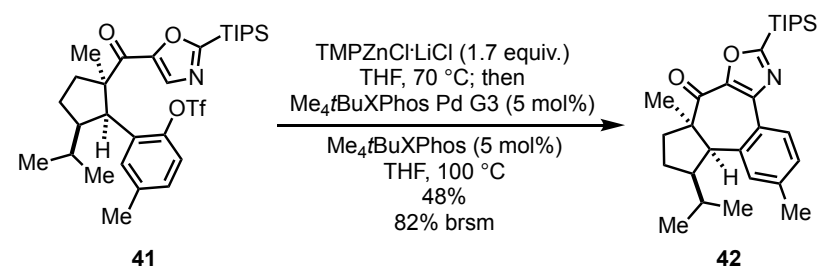

To each of five flame-dried microwave tubes were added compound $41(227.0 \mathrm{mg}, 0.369$ mmol, 1.0 equiv.). The reaction tubes were then sealed with Teflon-caps and THF $(0.2 \mathrm{~mL})$ was added to each to dissolve the substrate. Then TMPZnCl. LiCl $(0.57 \mathrm{~mL}, 1.1 \mathrm{M}$ in THF, $0.627 \mathrm{mmol}, 1.7$ equiv.) was added to each tube. The reactions were warmed up to $70{ }^{\circ} \mathrm{C}$ and stirred for $6.5 \mathrm{~h}$ before they were cooled down to room temperature. The reaction tubes were opened in the glovebox, and $\mathrm{Me}_{4} t \mathrm{BuXPhos}$ Pd G3 (19.3 mg, $0.0184 \mathrm{mmol}, 5 \mathrm{~mol} \%$ ) and $\mathrm{Me}_{4} t \mathrm{BuXPhos}(10.8 \mathrm{mg}, 0.0184 \mathrm{mmol}, 5 \mathrm{~mol} \%)$ were added to each of the mixtures. Then the reaction tubes were sealed again and the mixtures were stirred at $100{ }^{\circ} \mathrm{C}$ for $11 \mathrm{~h}$ before they were cooled down to room temperature and quenched with saturated aqueous ammonium chloride $(0.5 \mathrm{~mL}$ for each). All the residue of the five reactions were combined and extracted with ether $(20 \mathrm{~mL} \times 3)$. The combined organic phase was washed with brine $(50 \mathrm{~mL})$, dried $\left(\mathrm{Na}_{2} \mathrm{SO}_{4}\right)$, filtered and concentrated. The residue was purified on column chromatography (hexane/ether $=30 / 1$ to $4 / 1$ ). Compound 42 was obtained as a light-yellow oil (503.3 mg, 48\%, 82\% BRSM) and compound 41 (576.1 mg, 42\%) was recovered. ${ }^{1}$ H NMR (500 MHz, $\left.\mathbf{C D C l}_{3}\right) \delta 8.25(\mathrm{~d}, J=8.0 \mathrm{~Hz}, 1 \mathrm{H}), 7.19(\mathrm{~d}, J=8.0 \mathrm{~Hz}, 1 \mathrm{H}), 7.11(\mathrm{~s}, 1 \mathrm{H}), 3.26(\mathrm{~d}, J=10.2 \mathrm{~Hz}$, 1H), $2.86-2.76(\mathrm{~m}, 1 \mathrm{H}), 2.38(\mathrm{~s}, 3 \mathrm{H}), 2.15-2.07(\mathrm{~m}, 1 \mathrm{H}), 1.96-1.86(\mathrm{~m}, 1 \mathrm{H}), 1.54-1.43$ (m, 3H), $1.40-1.32(\mathrm{~m}, 2 \mathrm{H}), 1.22-1.11(\mathrm{~m}, 21 \mathrm{H}), 1.07-0.99(\mathrm{~m}, 1 \mathrm{H}), 0.51(\mathrm{~d}, J=6.6 \mathrm{~Hz}$, 
3H), $0.18(\mathrm{~d}, J=6.3 \mathrm{~Hz}, 3 \mathrm{H}) ;{ }^{13} \mathbf{C}$ NMR (125 MHz, $\left.\mathbf{C D C l}_{3}\right) \delta$ 192.1, 172.2, 145.4, 145.0, 139.7, 138.6, 134.1, 128.8, 128.1, 127.2, 59.7, 55.9, 51.3 38.2, 31.4, 28.4, 23.8, 23.0, 21.5, 21.4, 18.4, 11.0.; IR (film): 2951, 2925, 2868, 1658, 1463, 1380, 1366, 1018, $883 \mathrm{~cm}^{-1}$; HRMS (ESI): $m / z$ Calc. for $\mathrm{C}_{29} \mathrm{H}_{44} \mathrm{NO}_{2} \mathrm{Si}[\mathrm{M}+\mathrm{H}]^{+}: 466.3136$, found: 466.3135 .

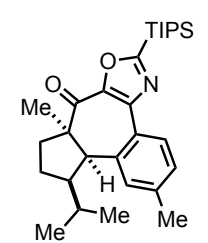

42

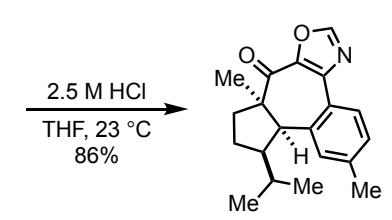

43

To the solution of compound 42 (1.17 g, $2.51 \mathrm{mmol}, 1.0$ equiv.) in THF (20 mL) was added aqueous $\mathrm{HCl}(8 \mathrm{~mL}, 2.5 \mathrm{M})$. The reaction mixture was stirred at room temperature for 1 day before it was diluted with water $(50 \mathrm{~mL})$ and extracted with ether $(50 \mathrm{~mL} \times 3)$. The combined organic phase was washed with saturated aqueous sodium bicarbonate $(50 \mathrm{~mL})$, brine $(50 \mathrm{~mL})$, dried $\left(\mathrm{Na}_{2} \mathrm{SO}_{4}\right)$, filtered and concentrated. The residue was purified by column chromatography (hexane/ether $=10 / 1$ to $6 / 1$ ). Compound $\mathbf{4 3}$ was obtained as a white solid $\left(0.67\right.$ g, 86\%). mp 113-114 ${ }^{\circ} \mathrm{C} ;{ }^{1} \mathbf{H}$ NMR (500 MHz, $\left.\mathbf{C D C l}_{3}\right) \delta 8.18(\mathrm{~d}, J=7.9 \mathrm{~Hz}, 1 \mathrm{H}), 8.02$ (s, 1H), $7.21(\mathrm{~d}, J=8.0 \mathrm{~Hz}, 1 \mathrm{H}), 7.14(\mathrm{~s}, 1 \mathrm{H}), 3.30(\mathrm{~d}, J=10.1 \mathrm{~Hz}, 1 \mathrm{H}), 2.86-2.77(\mathrm{~m}, 1 \mathrm{H})$, $2.38(\mathrm{~s}, 3 \mathrm{H}), 2.18-2.08(\mathrm{~m}, 1 \mathrm{H}), 1.96-1.86(\mathrm{~m}, 1 \mathrm{H}), 1.42-1.31(\mathrm{~m}, 2 \mathrm{H}), 1.16(\mathrm{~s}, 3 \mathrm{H}), 1.09$ - $1.00(\mathrm{~m}, 1 \mathrm{H}), 0.54(\mathrm{~d}, J=6.5 \mathrm{~Hz}, 3 \mathrm{H}), 0.16(\mathrm{~d}, J=6.3 \mathrm{~Hz}, 3 \mathrm{H}) ;{ }^{13} \mathbf{C}$ NMR (125 MHz, $\left.\mathbf{C D C l}_{3}\right) \delta 192.0,153.0,145.0,143.4,140.6,138.8,134.4,128.7,128.3,126.1,59.7,55.4$, 51.4, 37.7, 31.5, 28.4, 23.8, 23.1, 21.7, 21.4; IR (film): 2956, 2869, 1652, 1380, 1365, 1007 , 910, 829, $730 \mathrm{~cm}^{-1}$; HRMS (ESI): $\mathrm{m} / z$ Calc. for $\mathrm{C}_{20} \mathrm{H}_{24} \mathrm{NO}_{2}[\mathrm{M}+\mathrm{H}]^{+}: 310.1801$, found: 310.1800 . 


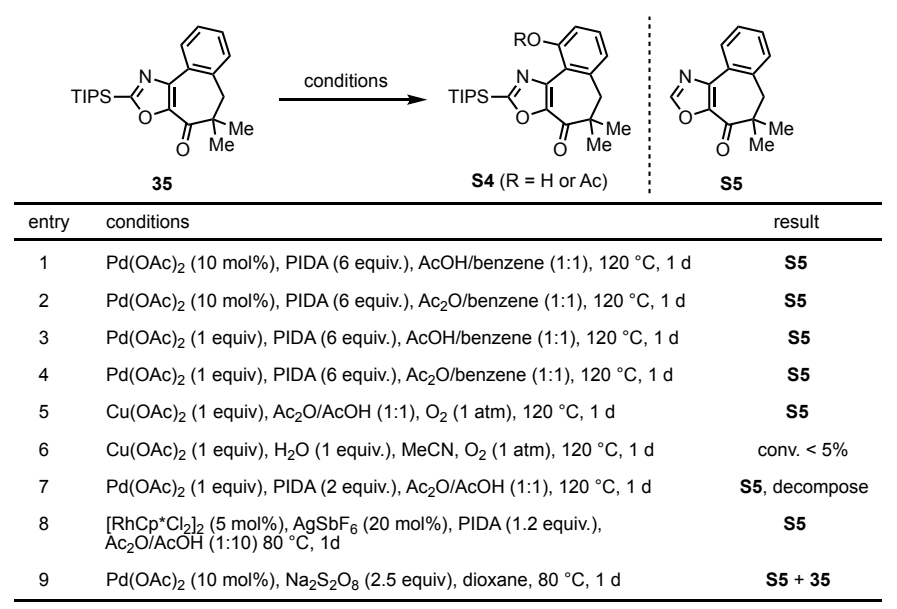

Table S3. Attempted C-H oxidation of $\mathbf{3 5}$

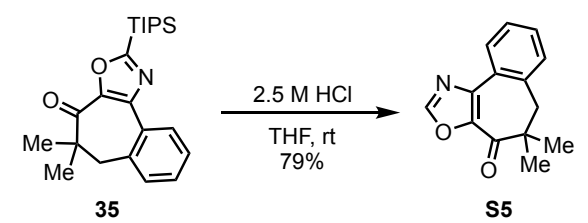

To a solution of 35 (3.63 g, $9.46 \mathrm{mmol}, 1.0$ equiv.) in THF (30 mL) was added aqueous $\mathrm{HCl}$ $(15 \mathrm{~mL}, 2.5 \mathrm{M})$. The reaction mixture was stirred at room temperature for $20 \mathrm{~h}$. Then the residue was diluted with water $(50 \mathrm{~mL})$ and extracted with ether $(50 \mathrm{~mL} \times 3)$. The combined organic phase was washed with saturated aqueous sodium bicarbonate $(50 \mathrm{~mL})$, brine $(50 \mathrm{~mL})$, dried $\left(\mathrm{Na}_{2} \mathrm{SO}_{4}\right)$, filtered and concentrated. The residue was purified by column chromatography (hexane/ether $=10 / 1$ to $5 / 1$ ). Compound S5 was obtained as white solid $(1.70 \mathrm{~g}, 79 \%) .{ }^{1} \mathbf{H}$ NMR (500 MHz, $\left.\mathbf{C D C l}_{3}\right) \delta=8.16(\mathrm{dd}, J=7.6,1.5 \mathrm{~Hz}, 1 \mathrm{H}), 8.09(\mathrm{~s}, 1 \mathrm{H})$, 7.44 (ddd, $J=7.6,7.5,1.5 \mathrm{~Hz}, 1 \mathrm{H}), 7.39$ (ddd, $J=7.5,7.5,1.5 \mathrm{~Hz}, 1 \mathrm{H}), 7.29$ (d, $J=7.5 \mathrm{~Hz}$, 1H), 3.03 (br, 2H), 1.15 (br, 6H); ${ }^{13} \mathbf{C}$ NMR (125 MHz, $\left.\mathbf{C D C l}_{3}\right) \delta=192.8,153.4,143.8$, 143.3, 137.3, 131.3, 130.2, 128.9, 127.9, 127.6, 43.9, 43.7; IR (film): 3122, 2966, 2929, 1663, 1563, 1515, 1386, 1361, 1226, 1024, 764, $704 \mathrm{~cm}^{-1}$; HRMS (ESI): $m / z$ Calc. for $\mathrm{C}_{14} \mathrm{H}_{14} \mathrm{NO}_{2}$ $[\mathrm{M}+\mathrm{H}]^{+}:$228.1019, found: 228.1020 . 


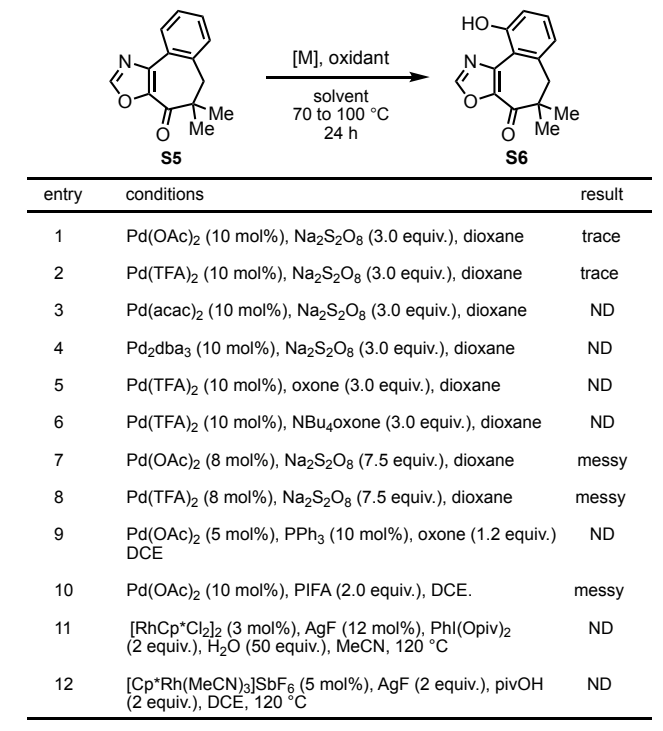

Table S4. Attempted C-H oxidation of S5

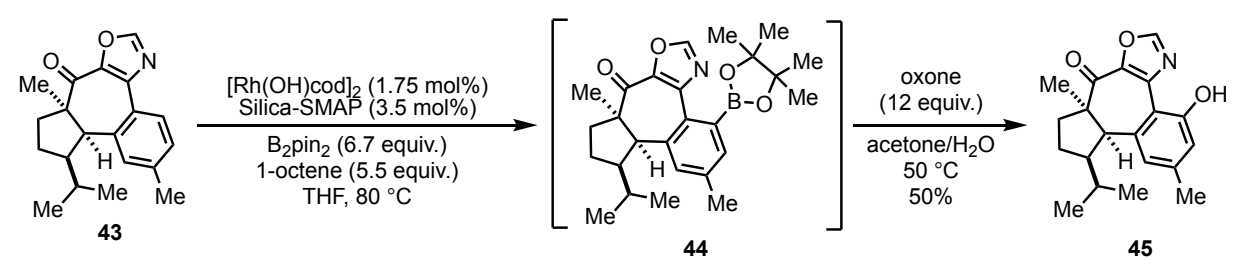

One-pot small-scale synthesis procedure: To compound 43 (25.5 mg, $0.0824 \mathrm{mmol}, 1.0$ equiv.), $\mathrm{B}_{2}$ pin $_{2}$ (87.9 mg, $0.346 \mathrm{mmol}, 4.2$ equiv.), Silica-SMAP (20.6 mg, $0.08 \mathrm{mmol} / \mathrm{g}$, $0.0016 \mathrm{mmol}, 2 \mathrm{~mol} \%)$, 1-octene ( $40 \mu \mathrm{L}, 0.25 \mathrm{mmol}, 3$ equiv.) and THF ( $0.1 \mathrm{~mL})$ was added a solution of $[\mathrm{Rh}(\mathrm{OH}) \mathrm{cod}]_{2}(0.4 \mathrm{mg}, 0.0008 \mathrm{mmol}, 1 \mathrm{~mol} \%)$ in THF $(0.1 \mathrm{~mL})$. The mixture was stirred at $80{ }^{\circ} \mathrm{C}$ for $4.5 \mathrm{~h}$ before it was cooled down to room temperature and more $[\mathrm{Rh}(\mathrm{OH}) \operatorname{cod}]_{2}(0.19 \mathrm{mg}, 0.0004 \mathrm{mmol}, 0.5 \mathrm{~mol} \%)$, Silica-SMAP (10.3 mg, $0.08 \mathrm{mmol} / \mathrm{g}$, $0.0008 \mathrm{mmol}, 1 \mathrm{~mol} \%), \mathrm{B}_{2} \operatorname{pin}_{2}(31.4 \mathrm{mg}, 0.123 \mathrm{mmol}, 1.5$ equiv.) and 1-octene (20 $\mu \mathrm{L}, 0.12$ mmol, 1.5 equiv.) were added. Then the mixture was warmed up to $80^{\circ} \mathrm{C}$ and stirred for 11.5 $\mathrm{h}$ before it was cooled down to room temperature again and more $[\mathrm{Rh}(\mathrm{OH}) \operatorname{cod}]_{2}(0.1 \mathrm{mg}$, $0.0002 \mathrm{mmol}, 0.25 \mathrm{~mol} \%$ ), Silica-SMAP (5.2 mg, $0.08 \mathrm{mmol} / \mathrm{g}, 0.0004 \mathrm{mmol}, 0.5 \mathrm{~mol} \%$ ), $\mathrm{B}_{2} \operatorname{pin}_{2}(21.0 \mathrm{mg}, 0.0827 \mathrm{mmol}, 1$ equiv.) and 1-octene (13 $\mu \mathrm{L}, 0.08 \mathrm{mmol}, 1.0$ equiv.) were added. The reaction was warmed up to $80^{\circ} \mathrm{C}$ again and further stirred for $12.5 \mathrm{~h}$ before it was cooled down to room temperature and poured into water $(3 \mathrm{~mL})$ and acetone $(1.5 \mathrm{~mL})$. Oxone (300 $\mathrm{mg}, 0.98 \mathrm{mmol}, 12$ equiv.) was then added to the reaction mixture. The reaction was warmed up to $50{ }^{\circ} \mathrm{C}$ and stirred for $10 \mathrm{~h}$ before it was cooled down to room temperature, 
diluted with water $(1 \mathrm{~mL})$ and ether $(3 \mathrm{~mL})$, and extracted with ether $(3 \mathrm{~mL} \times 3)$. The combined organic phase was washed with water $(10 \mathrm{~mL})$, aqueous $10 \%$ sodium thiosulfate $(10 \mathrm{~mL})$ and brine $(10 \mathrm{~mL})$, dried $\left(\mathrm{Na}_{2} \mathrm{SO}_{4}\right)$, filtered and concentrated. The residue was purified on column chromatography (hexane/ether $=6 / 1$ ). Compound 45 was obtained as a white solid (13.3 mg, 50\%). mp 131-132 ${ }^{\circ} \mathrm{C} ;{ }^{1} \mathbf{H}$ NMR (500 MHz, $\left.\mathbf{C D C l}_{3}\right) \delta 11.65(\mathrm{~s}, 1 \mathrm{H})$, $8.17(\mathrm{~s}, 1 \mathrm{H}), 6.79(\mathrm{~s}, 1 \mathrm{H}), 6.68(\mathrm{~s}, 1 \mathrm{H}), 3.30(\mathrm{~d}, J=10.2 \mathrm{~Hz}, 1 \mathrm{H}), 2.86-2.75(\mathrm{~m}, 1 \mathrm{H}), 2.32(\mathrm{~s}$, $3 \mathrm{H}), 2.17-2.04(\mathrm{~m}, 1 \mathrm{H}), 1.99-1.88(\mathrm{~m}, 1 \mathrm{H}), 1.43-1.29(\mathrm{~m}, 2 \mathrm{H}), 1.18(\mathrm{~s}, 3 \mathrm{H}), 1.10-1.00$ $(\mathrm{m}, 1 \mathrm{H}), 0.56(\mathrm{~d}, J=6.5 \mathrm{~Hz}, 3 \mathrm{H}), 0.23(\mathrm{~d}, J=6.3 \mathrm{~Hz}, 3 \mathrm{H}) ;{ }^{13} \mathbf{C}$ NMR (125 MHz, CDCl$) \delta$ $191.7,158.7,151.5,143.9,143.0,142.8,139.8,126.6,117.6,110.4,60.0,55.2,51.6,37.8$, 31.7, 28.7, 23.5, 23.1, 21.9, 21.4; IR (film): 2954, 2922, 2851, 1585, 1462, 1377, 1377, 1264 , $739 \mathrm{~cm}^{-1}$; HRMS (ESI): $\mathrm{m} / z$ Calc. for $\mathrm{C}_{20} \mathrm{H}_{24} \mathrm{NO}_{3}[\mathrm{M}+\mathrm{H}]^{+}: 326.1750$, found: 326.1750 .

Note: For large scale, one-pot procedure would cause difficult isolation and low yield. To avoid this problem, a stepwise procedure was used as described below.

Two-step scaled-up synthesis procedure: To compound $\mathbf{4 3}$ (412.6 mg, $1.33 \mathrm{mmol}, 1.0$ equiv.), $\mathrm{B}_{2} \operatorname{pin}_{2}(1.42 \mathrm{~g}, 5.59 \mathrm{mmol}, 4.2$ equiv.), Silica-SMAP (0.33 g, $0.08 \mathrm{mmol} / \mathrm{g}, 0.026 \mathrm{mmol}, 2$ mol\%), 1-octene (630 $\mu \mathrm{L}, 4.0 \mathrm{mmol}, 3.0$ equiv.) and $[\mathrm{Rh}(\mathrm{OH}) \operatorname{cod}]_{2}(6.1 \mathrm{mg}, 0.013 \mathrm{mmol}, 1$ mol\%) was added THF $(3.2 \mathrm{~mL})$. The reaction was stirred at $80{ }^{\circ} \mathrm{C}$ for $9 \mathrm{~h}$. The mixture was cooled down to room temperature and more $[\mathrm{Rh}(\mathrm{OH}) \operatorname{cod}]_{2}(3.0 \mathrm{mg}, 0.0066 \mathrm{mmol}, 0.5 \mathrm{~mol} \%)$, Silica-SMAP (0.17 g, $0.08 \mathrm{mmol} / \mathrm{g}, 0.013 \mathrm{mmol}, 1 \mathrm{~mol} \%), \mathrm{B}_{2} \mathrm{pin}_{2}(0.51 \mathrm{~g}, 2.0 \mathrm{mmol}, 1.5$ equiv.) and 1-octene (310 $\mu \mathrm{L}, 2.0 \mathrm{mmol}, 1.5$ equiv.) were added. The mixture was warmed up to $80{ }^{\circ} \mathrm{C}$ and stirred for $10 \mathrm{~h}$ before it was cooled down to room temperature again and more $[\mathrm{Rh}(\mathrm{OH}) \mathrm{cod}]_{2}(1.5 \mathrm{mg}, 0.0033 \mathrm{mmol}, 0.25 \mathrm{~mol} \%)$, Silica-SMAP (83.3 mg, $0.008 \mathrm{mmol} / \mathrm{g}$, $0.0066 \mathrm{mmol}, 0.5 \mathrm{~mol} \%), \mathrm{B}_{2} \operatorname{pin}_{2}(0.34 \mathrm{~g}, 1.33 \mathrm{mmol}, 1.0$ equiv.) and 1-octene (210 $\mu \mathrm{L}, 1.34$ mmol, 1.0 equiv.) were added. The mixture was warmed up to $80^{\circ} \mathrm{C}$ again and further stirred for $9.5 \mathrm{~h}$ before it was cooled down to room temperature and filtered through celite. The reaction vial was washed with ether and filtered, and the combined organic residue was concentrated and then purified on column chromatography (hexane/ether $=6 / 1)$. Compound 44 was obtained as a yellow solid (281.8 mg, 49\%). ${ }^{1}$ H NMR (500 MHz, $\left.\mathbf{C D C l}_{3}\right) \delta 7.96$ (s, 1H), $7.24(\mathrm{~s}, 1 \mathrm{H}), 7.13(\mathrm{~s}, 1 \mathrm{H}), 3.29(\mathrm{~d}, J=10.2 \mathrm{~Hz}, 1 \mathrm{H}), 2.85-2.77(\mathrm{~m}, 1 \mathrm{H}), 2.37$ (s, 3H), $2.17-2.07(\mathrm{~m}, 1 \mathrm{H}), 1.96-1.86(\mathrm{~m}, 1 \mathrm{H}), 1.46-1.41(\mathrm{~m}, 12 \mathrm{H}), 1.38-1.33(\mathrm{~m}, 2 \mathrm{H}), 1.18(\mathrm{~s}$, 
3H), $1.07-0.97(\mathrm{~m}, 1 \mathrm{H}), 0.51(\mathrm{~d}, J=6.5 \mathrm{~Hz}, 3 \mathrm{H}), 0.15(\mathrm{~d}, J=6.3 \mathrm{~Hz}, 3 \mathrm{H}) ;{ }^{13} \mathbf{C}$ NMR (125 MHz, $\left.\mathbf{C D C l}_{3}\right) \delta 191.9,151.9,145.7,143.0,139.8,138.8,135.0,132.5,128.4,83.8,60.0,55.5$, 51.4, 37.7, 31.5, 28.3, 24.9, 24.5, 23.7, 23.4, 21.7, 21.3; IR (film): 2974, 2870, 2030, 1659, 1594, 1467, 1374, 1348, 1296, $1145 \mathrm{~cm}^{-1}$; HRMS (ESI): $m / z$ Calc. for $\mathrm{C}_{26} \mathrm{H}_{35} \mathrm{BNO}_{4}[\mathrm{M}+\mathrm{H}]^{+}$: 436.2658, found: 436.2660 .

To compound 44 (281.8 mg, $0.647 \mathrm{mmol}, 1.0$ equiv.) dissolved in acetone (16 mL) was added oxone ( $0.50 \mathrm{~g}, 1.6 \mathrm{mmol}, 2.5$ equiv.) dissolved in $\mathrm{H}_{2} \mathrm{O}(8 \mathrm{~mL})$. The reaction was heated to 50 ${ }^{\circ} \mathrm{C}$ and stirred for $7.5 \mathrm{~h}$ before it was cooled down to room temperature, diluted with water (20 $\mathrm{mL})$ and extracted with ether $(20 \mathrm{~mL} \times 3)$. The combined organic phase was washed with water, aqueous $10 \%$ sodium thiosulfate and brine, dried $\left(\mathrm{Na}_{2} \mathrm{SO}_{4}\right)$, filtered and concentrated. The residue was purified on column chromatography (hexane/ether $=6 / 1$ ). Compound $\mathbf{4 5}$ was obtained as white solid (180.0 mg, 86\%; $42 \%$ from $\mathbf{4 3}$ for two steps).

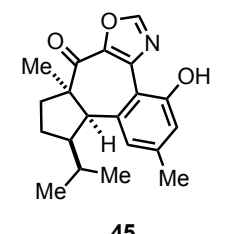

45

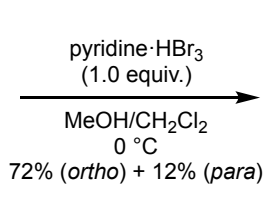

(20.9)

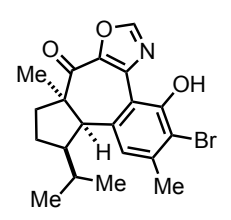

Hamigeran M (6)

To compound 45 (90.0 mg, 0.277 mmol, 1.0 equiv.) in a co-solvent ( $3 \mathrm{~mL}, \mathrm{DCM} / \mathrm{MeOH}=5 / 2)$ at $0{ }^{\circ} \mathrm{C}$ was added pyridine $\cdot \mathrm{HBr}_{3}(98.3 \mathrm{mg}, 90 \% \mathrm{w} / \mathrm{w}, 0.277 \mathrm{mmol}, 1.0$ equiv.) dissolved in the same co-solvent $(1.2 \mathrm{~mL})$ during $15 \mathrm{~min}$. The mixture was further stirred for $15 \mathrm{~min}$ at 0 ${ }^{\circ} \mathrm{C}$ and then poured into ether $(20 \mathrm{~mL})$ and water $(40 \mathrm{~mL})$. The residue was extracted with ether $(20 \mathrm{~mL} \times 3)$, washed with saturated sodium bicarbonate $(30 \mathrm{~mL})$ and brine $(30 \mathrm{~mL})$, dried $\left(\mathrm{Na}_{2} \mathrm{SO}_{4}\right)$, filtered and concentrated. The residue was purified on column chromatography (hexane/EtOAc $=20 / 1)$. Pure compound 6 was obtained as white solid $(67.4$ $\mathrm{mg}, 60 \%)$ together with a mixture of ortho- and para- brominated products $(26.6 \mathrm{mg}$, ortho/para $=1: 1,24 \%$ ). The latter mixture was further separated using preparative TLC with hexane/toluene/ether (20/20/1). The $20 \mathrm{~cm}$ x $20 \mathrm{~cm}$ TLC plate was developed three times with the above eluent to ensure a good separation. Overall, hamigeran M (6) was obtained in $72 \%$ yield $(81 \mathrm{mg})$ in one batch with $12 \%$ of the para-brominated isomer. This reaction was repeated one more time. Together with the previous smaller scale investigations, we have 
accumulated over $200 \mathrm{mg}$ racemic hamigeran M. mp 155-156 ${ }^{\circ} \mathrm{C} ;{ }^{1} \mathbf{H}$ NMR (500 MHz, $\left.\mathbf{C D C l}_{3}\right) \delta 12.55(\mathrm{~s}, 1 \mathrm{H}), 8.22(\mathrm{~s}, 1 \mathrm{H}), 6.80(\mathrm{~s}, 1 \mathrm{H}), 3.28(\mathrm{~d}, J=10.2 \mathrm{~Hz}, 1 \mathrm{H}), 2.84-2.76(\mathrm{~m}$, 1H), $2.44(\mathrm{~s}, 3 \mathrm{H}), 2.17-2.07(\mathrm{~m}, 1 \mathrm{H}), 1.98-1.89(\mathrm{~m}, 1 \mathrm{H}), 1.43-1.31(\mathrm{~m}, 2 \mathrm{H}), 1.17(\mathrm{~s}, 3 \mathrm{H})$, 1.04 (dqq, $J=10.1,6.4,6.4 \mathrm{~Hz}, 1 \mathrm{H}), 0.57(\mathrm{~d}, J=6.4 \mathrm{~Hz}, 3 \mathrm{H}), 0.25(\mathrm{~d}, J=6.4 \mathrm{~Hz}, 3 \mathrm{H}) ;{ }^{13} \mathbf{C}$ NMR (125 MHz, CDCl $) \delta 191.4,155.1,151.4,143.3,142.9,142.5,138.5,127.1,113.5$, 111.5, 59.8, 55.2, 51.6, 37.7, 31.7, 28.5, 23.6, 23.4, 23.3, 21.8; IR (film): 3132, 2956, 2868 , 1659, 1583, 1382, 1191, 964, $732 \mathrm{~cm}^{-1}$; HRMS (ESI): $m / z$ Calc. for $\mathrm{C}_{20} \mathrm{H}_{23} \mathrm{NO}_{3} \mathrm{Br}[\mathrm{M}+\mathrm{H}]^{+}$: 404.0856/404.0837, found: 404.0854/404.0836. 
Table S5. ${ }^{1} \mathrm{H}$ NMR and ${ }^{13} \mathrm{C}$ NMR comparasion of synthetic Hamigeran $\mathrm{M}$ and natural Hamigeran M. ${ }^{[6]}$

\begin{tabular}{|c|c|c|c|c|c|}
\hline \multirow{2}{*}{ position } & \multicolumn{2}{|c|}{${ }^{1} \mathrm{H}$} & \multicolumn{3}{|c|}{${ }^{13} \mathrm{C}$} \\
\hline & natural & synthesized & natural & synthesized & $\Delta_{\text {nat-syn }}$ \\
\hline 1 & & & 155.2 & 155.1 & 0.1 \\
\hline $1-\mathrm{OH}$ & 12.57 & 12.55 & & & \\
\hline 2 & & & 113.6 & 113.5 & 0.1 \\
\hline 3 & & & 142.7 & 142.5 & 0.2 \\
\hline 4 & 6.80 & 6.8 & 127.2 & 127.2 & 0 \\
\hline $4 a$ & & & 138.6 & 138.5 & 0.1 \\
\hline 5 & 3.28 & 3.28 & 59.9 & 59.8 & 0.1 \\
\hline 6 & 2.12 & 2.12 & 51.6 & 51.6 & 0 \\
\hline \multirow[t]{2}{*}{7} & 1.35 & 1.36 & 28.7 & 28.5 & 0.2 \\
\hline & 1.93 & 1.94 & & & \\
\hline \multirow[t]{2}{*}{8} & 1.35 & 1.36 & 37.8 & 37.7 & 0.1 \\
\hline & 2.79 & 2.79 & & & \\
\hline 9 & & & 55.3 & 55.2 & 0.1 \\
\hline 10 & & & 191.6 & 191.4 & 0.2 \\
\hline 11 & & & 143.4 & 143.3 & 0.1 \\
\hline 12 & & & 143.1 & 142.9 & 0.2 \\
\hline $12 \mathrm{a}$ & & & 111.6 & 111.5 & 0.1 \\
\hline 13 & 1.03 & 1.04 & 31.9 & 31.7 & 0.2 \\
\hline 14 & 0.56 & 0.57 & 21.9 & 21.8 & 0.1 \\
\hline 15 & 0.24 & 0.25 & 23.4 & 23.3 & 0.1 \\
\hline 16 & 1.17 & 1.17 & 23.5 & 23.4 & 0.1 \\
\hline 17 & 2.44 & 2.44 & 23.8 & 23.6 & 0.2 \\
\hline 18 & 8.22 & 8.22 & 151.5 & 151.4 & 0.1 \\
\hline
\end{tabular}

Part 2. X-ray structure and analysis data<smiles>Cc1cc2c(c(O)c1Br)-c1ncoc1C(=O)[C@@]1(C)CCC(C(C)C)[C@]21C</smiles>

Hamigeran M (6)

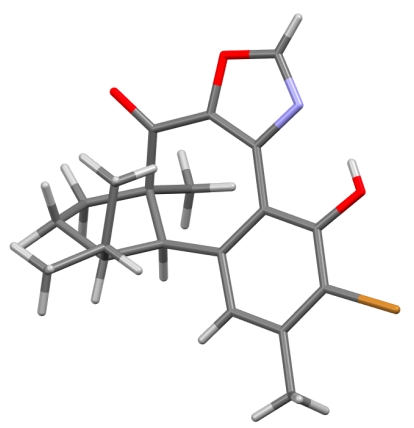

Figure S1. X-ray structure of hamigeran M (6)

A colorless plate-shaped crystal of synthetic hamigeran M for X-ray diffraction was obtained 
by crystallization in a two-layer mixture in $\mathrm{DCM} / \mathrm{methanol}$. Hamigeram $\mathrm{M}(6,5 \mathrm{mg})$ was dissolved in 2 drops of DCM. Then $\mathrm{MeOH}(0.2 \mathrm{ml})$ was slowly added to form a 2-layer mixture, which was stored at room temperature overnight to afford the desired crystal. The data were collected at 150(2) K on a Bruker AXS D8 Quest CMOS diffractometer with Mo sealed tube and curved triumph monochromator with a $10 \mathrm{~cm}$ x $10 \mathrm{~cm}$ Photon-100 detector and fixed chi angle. The supplementary crystallographic data was deposited in The Cambridge Crystallographic Data Centre. CCDC 2114503.

\section{X-ray analysis data:}

Bond precision: $\mathrm{C}-\mathrm{C}=0.0040$ A Wavelength $=1.54178$

Cell: $\mathrm{a}=6.6095(4) \mathrm{b}=9.4129(4) \mathrm{c}=14.3705(5)$

alpha $=91.154(2)$ beta $=90.449(2)$ gamma $=99.650(3)$

Temperature: $150 \mathrm{~K}$

Calculated Reported

Volume 881.17(7) 881.17(7)

Space group P -1 P -1

Hall group -P 1 -P 1

Moiety formula $\mathrm{C} 20 \mathrm{H} 22 \mathrm{Br} \mathrm{N} \mathrm{O} 3$ ?

Sum formula C20 H22 Br N O3 C20 H22 Br N O3

Mr 404.29404 .29

Dx,g cm-3 1.5241 .524

\section{Z 22}

$\mathrm{Mu}(\mathrm{mm}-1) 3.3363 .336$

F000 416.0416 .0

F000' 415.68

$\mathrm{h}, \mathrm{k}, \operatorname{lmax} 8,11,188,11,18$

Nref 38293669

Tmin,Tmax 0.532,0.627 0.414,0.754

Tmin' 0.374

Correction method $=\#$ Reported T Limits: $T \min =0.414$ Tmax $=0.754$ 
AbsCorr $=$ MULTI-SCAN

Data completeness $=0.958$ Theta $(\max )=79.609$

$\mathrm{R}($ reflections $)=0.0423(3568)$

$w R 2($ reflections $)=0.1292(3669)$

$\mathrm{S}=1.150 \mathrm{Npar}=234$

\section{Part 3. References}

(1) Sumida, Y.; Harada, R.; Kato-Sumida, T.; Johmoto, K.; Uekusa, H.; Hosoya, T. Boron-Selective Biaryl Coupling Approach to Versatile Dibenzoxaborins and Application to Concise Synthesis of Defucogilvocarcin M. Org. Lett. 2014, 16, 6240-6243.

(2) Miller, R. A.; Smith, R. M.; Marcune, B. A Convenient Oxazole C-2 Protecting Group: The Synthesis of 4- and 5-Substituted Oxazoles via Metalation of 2-Triisopropylsilyloxazoles. J. Org. Chem. 2005, 70, 9074-9076.

(3) Haas, D.; Mosrin, M.; Knochel, P. Regioselective Functionalization of the Oxazole Scaffold Using TMP-Bases of Mg and Zn. Org. Lett. 2013, 15, 6162-6165.

(4) Abraham, E.; Claridge, T. D. W.; Davies, S. G.; Odell, B.; Roberts, P. M.; Russell, A. J.; Smith, A. D.; Smith, L. J.; Storr, H. R.; Sweet, M. J.; Thompson, A. L.; Thomson, J. E.; Tranter, G. E.; Watkin, D, J. A systematic study of the solid state and solution phase conformational preferences of $\beta$-peptides derived from $\mathrm{C}(3)$-alkyl substituted transpentacin derivatives Tetrahedron: Asymmetry. 2011, 22, 69-100.

(5) Chattopadhyay, B.; Dannatt, J. E.; Andujar-De Sanctis, I. L.; Gore, K. A.; Maleczka, R. E.; Singleton, D. A.; Smith, M. R. Ir-Catalyzed ortho-Borylation of Phenols Directed by Substrate-Ligand Electrostatic Interactions: A Combined Experimental/in Silico Strategy for Optimizing Weak Interactions. J. Am. Chem. Soc. 2017, 139, 7864-7871.

(6) Dattelbaum, J. D.; Singh, A. J.; Field, J. J.; Miller, J. H.; Northcote, P. T. The Nitrogenous Hamigerans: Unusual Amino Acid-Derivatized Aromatic Diterpenoid Metabolites from the New Zealand Marine Sponge Hamigera tarangaensis. J. Org. Chem. 2015, 80, 304-312. 


\section{Part 4. ${ }^{1} \mathrm{H}$ and ${ }^{13} \mathrm{C}$ NMR spectra}
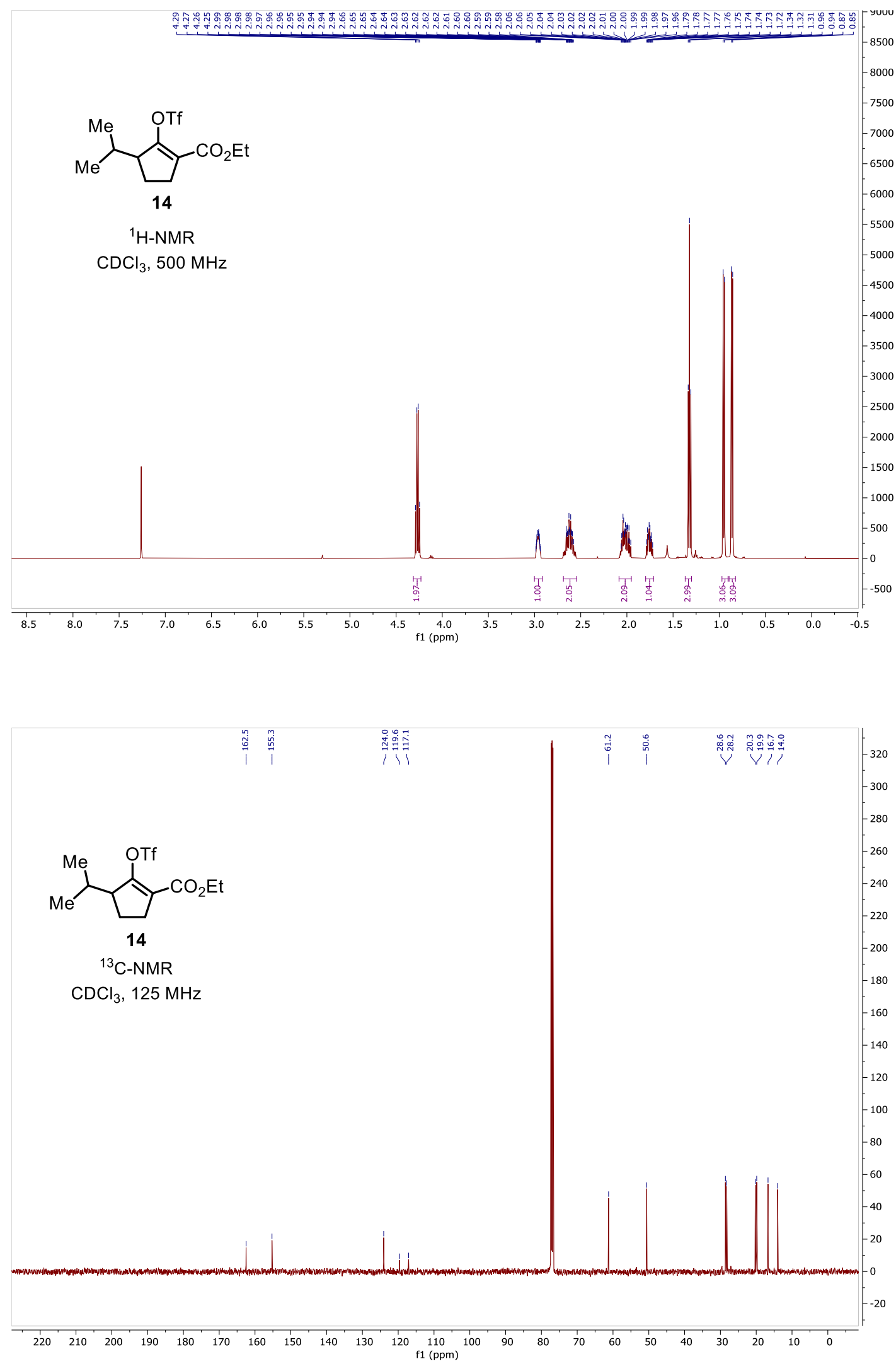


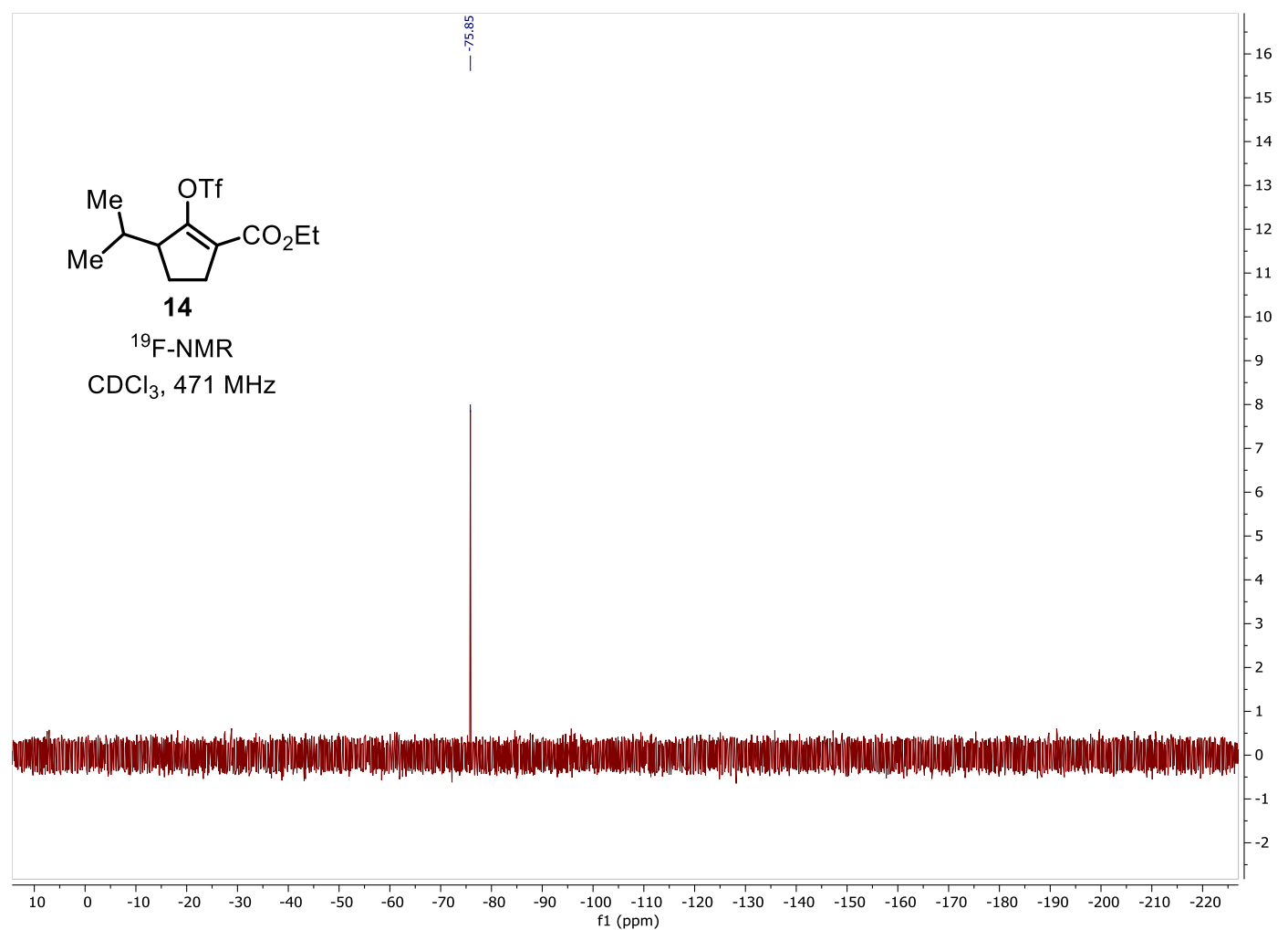



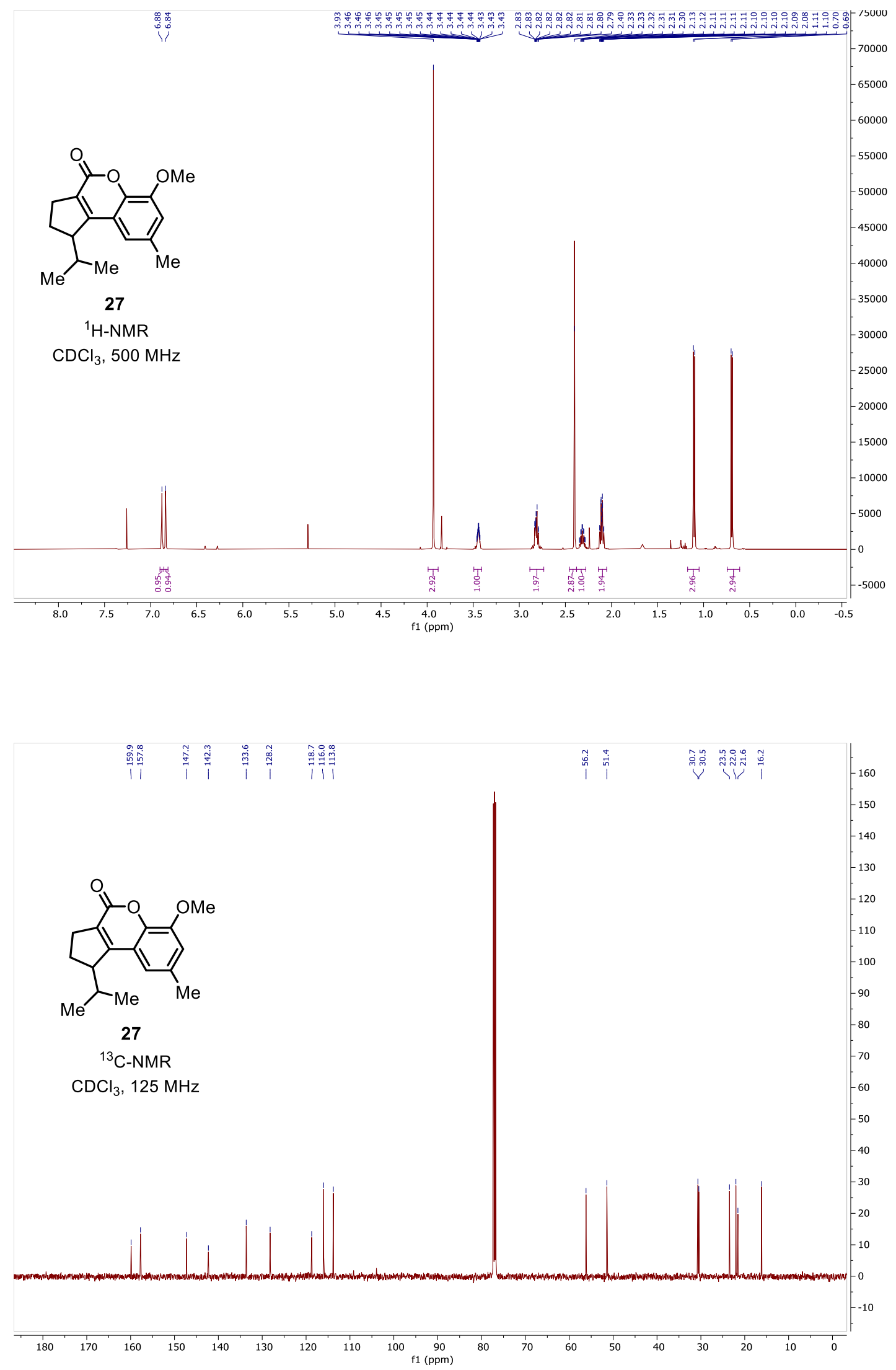

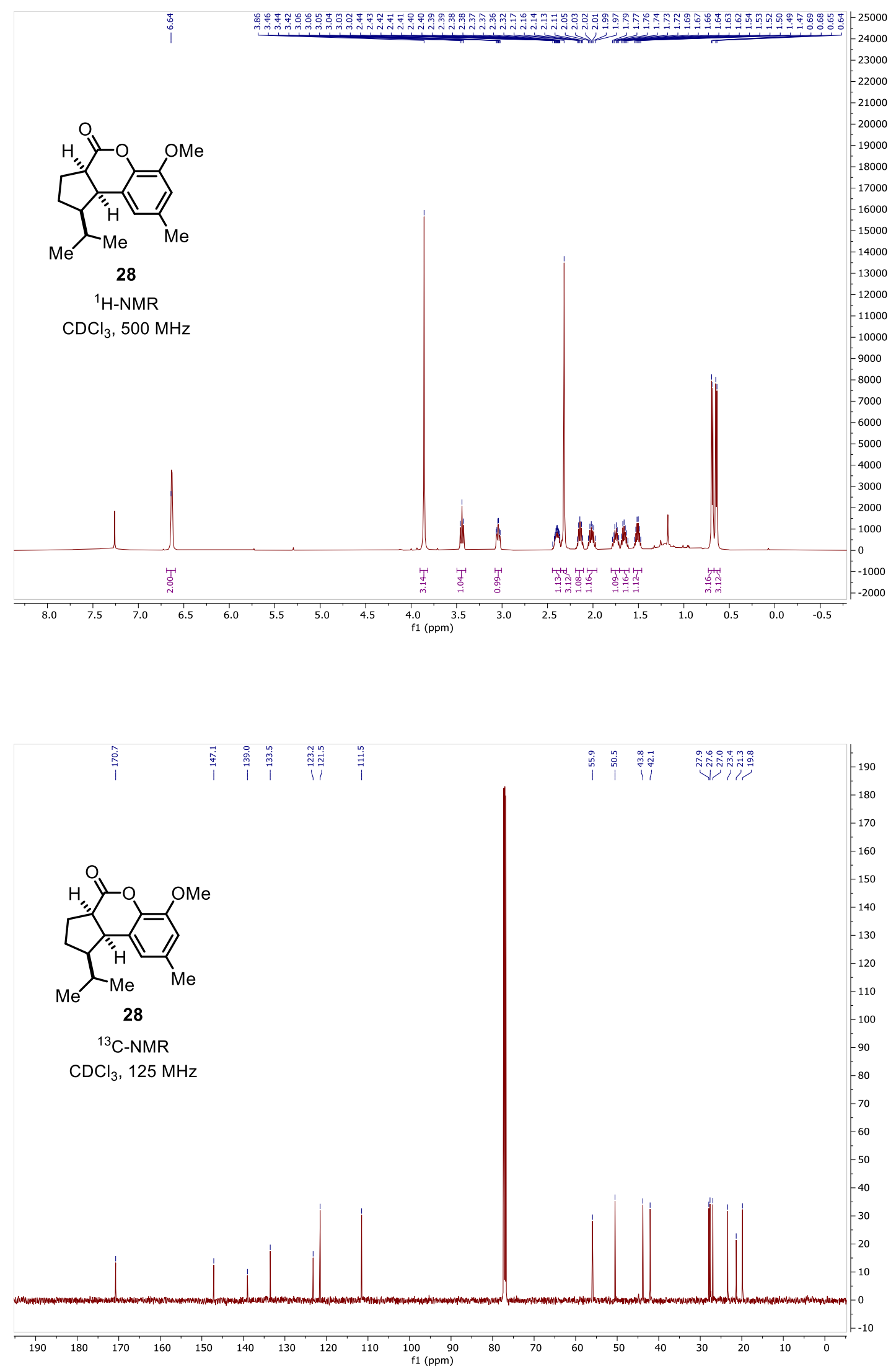

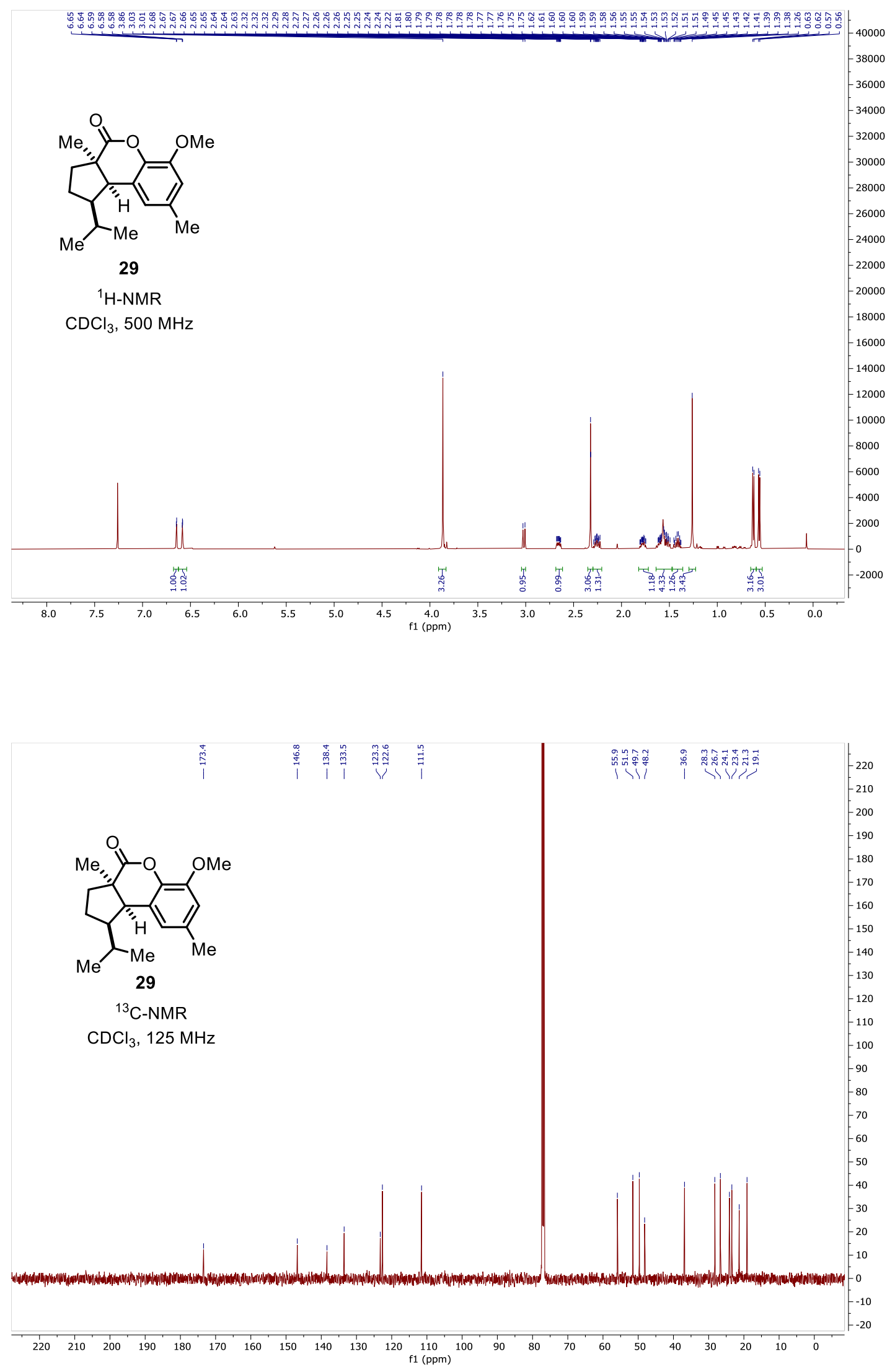

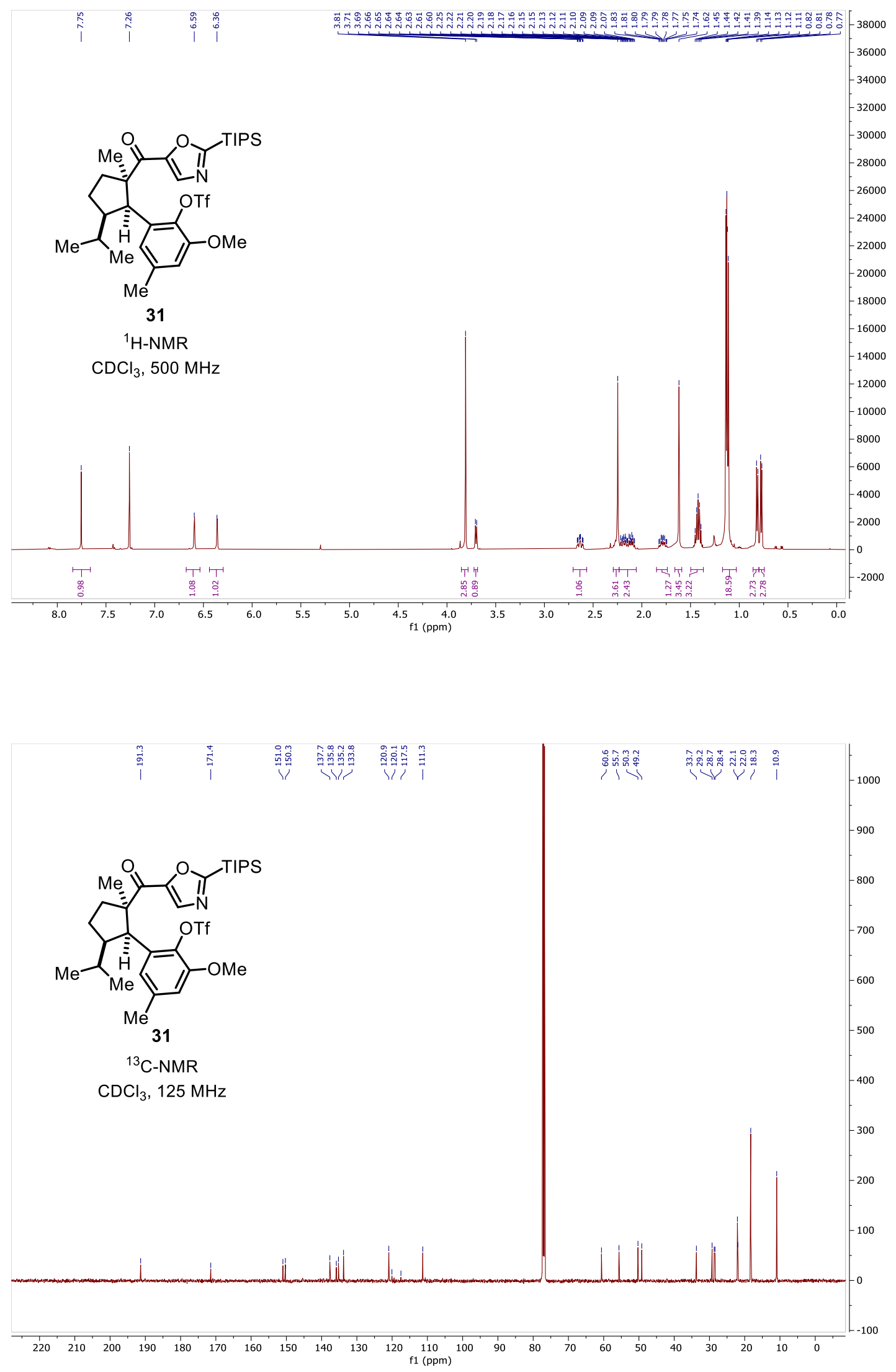


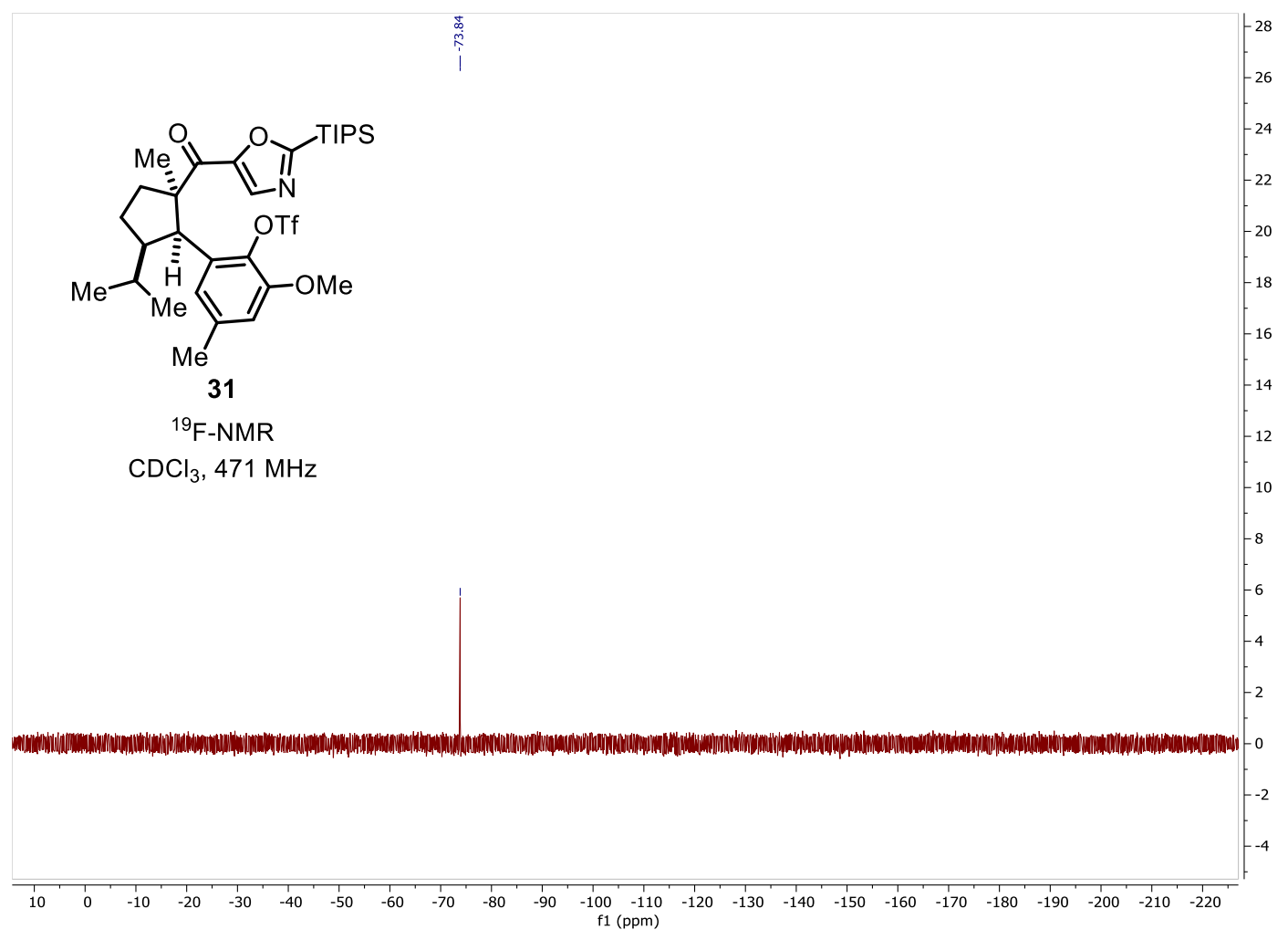




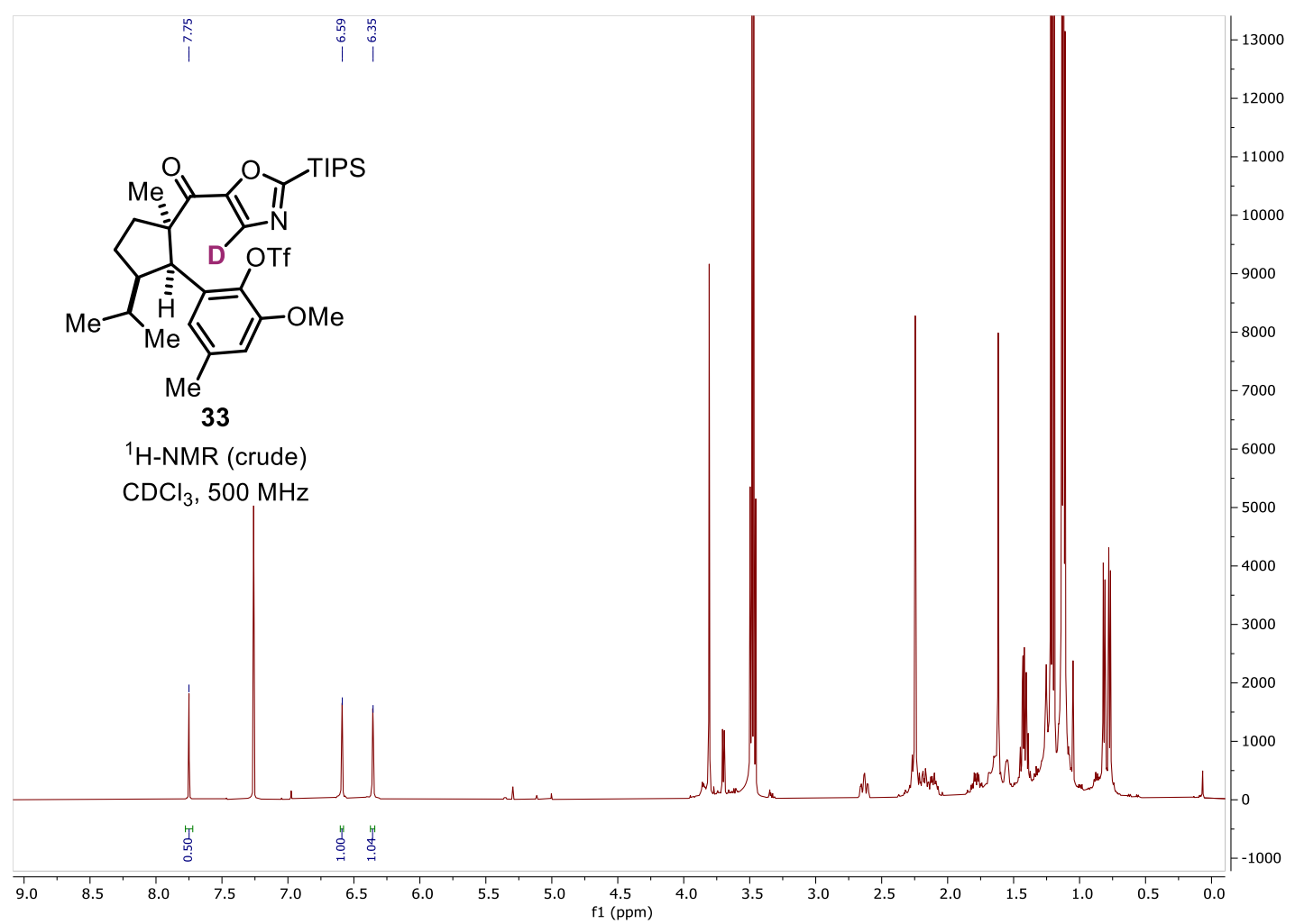



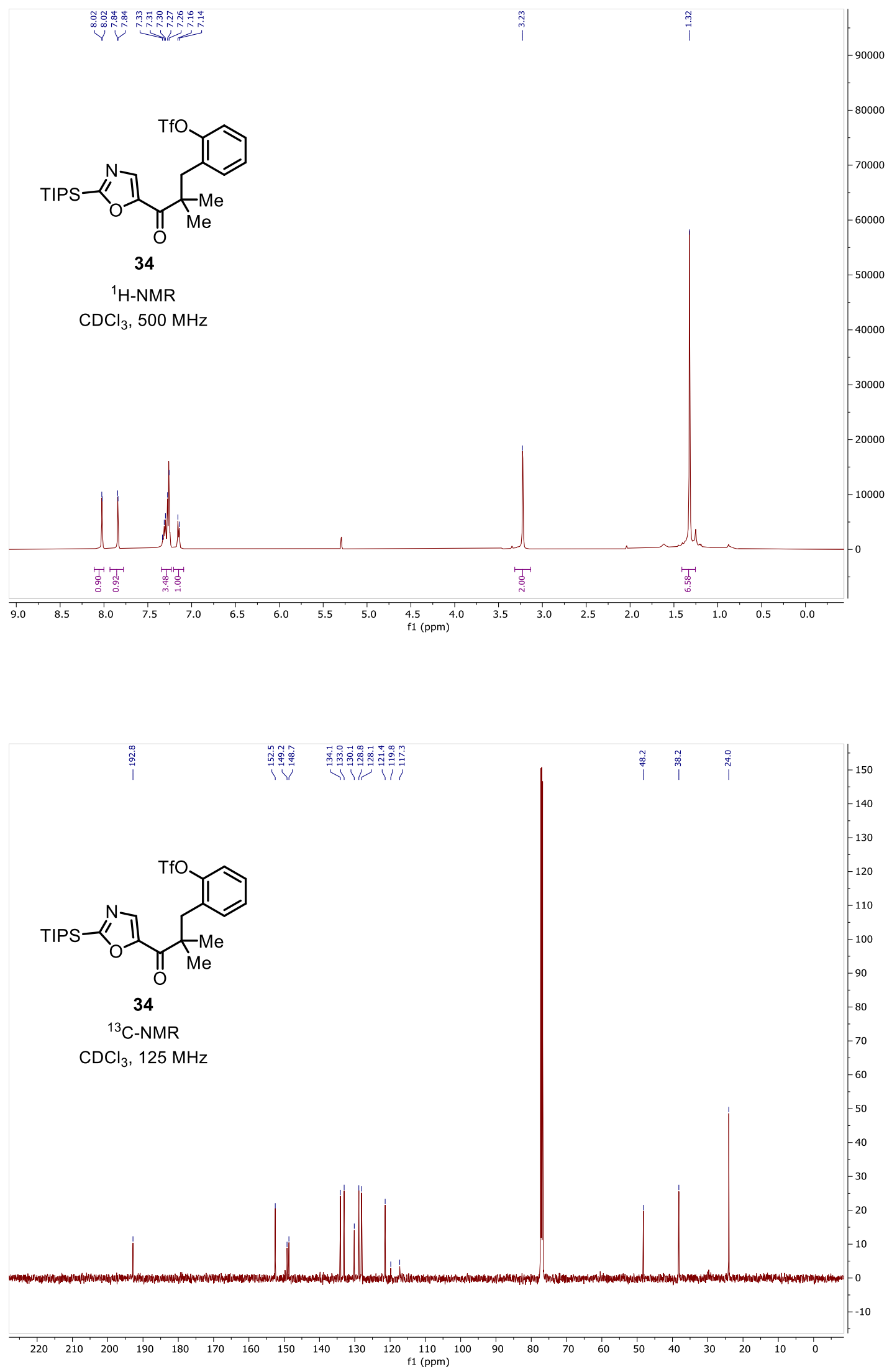


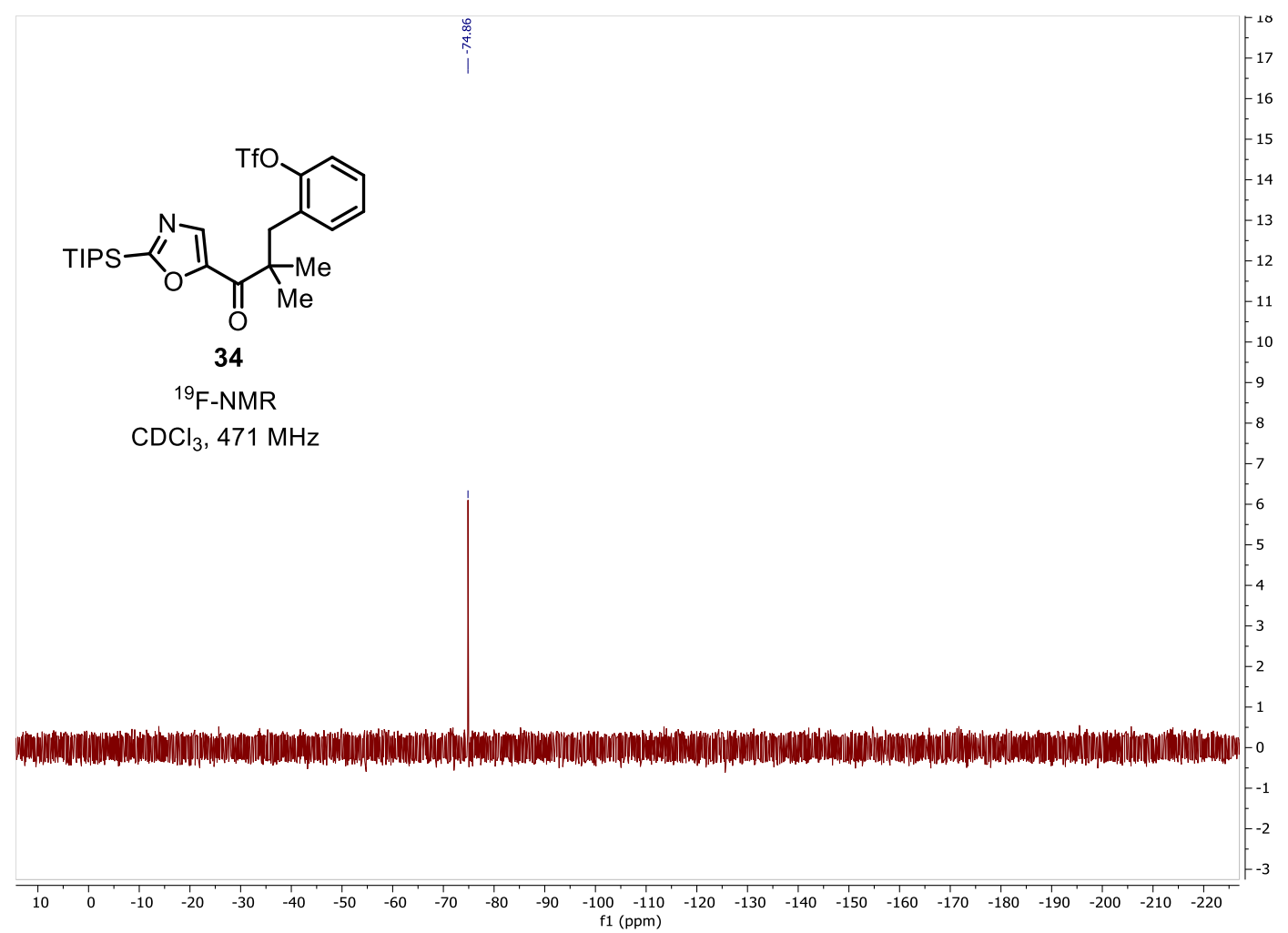



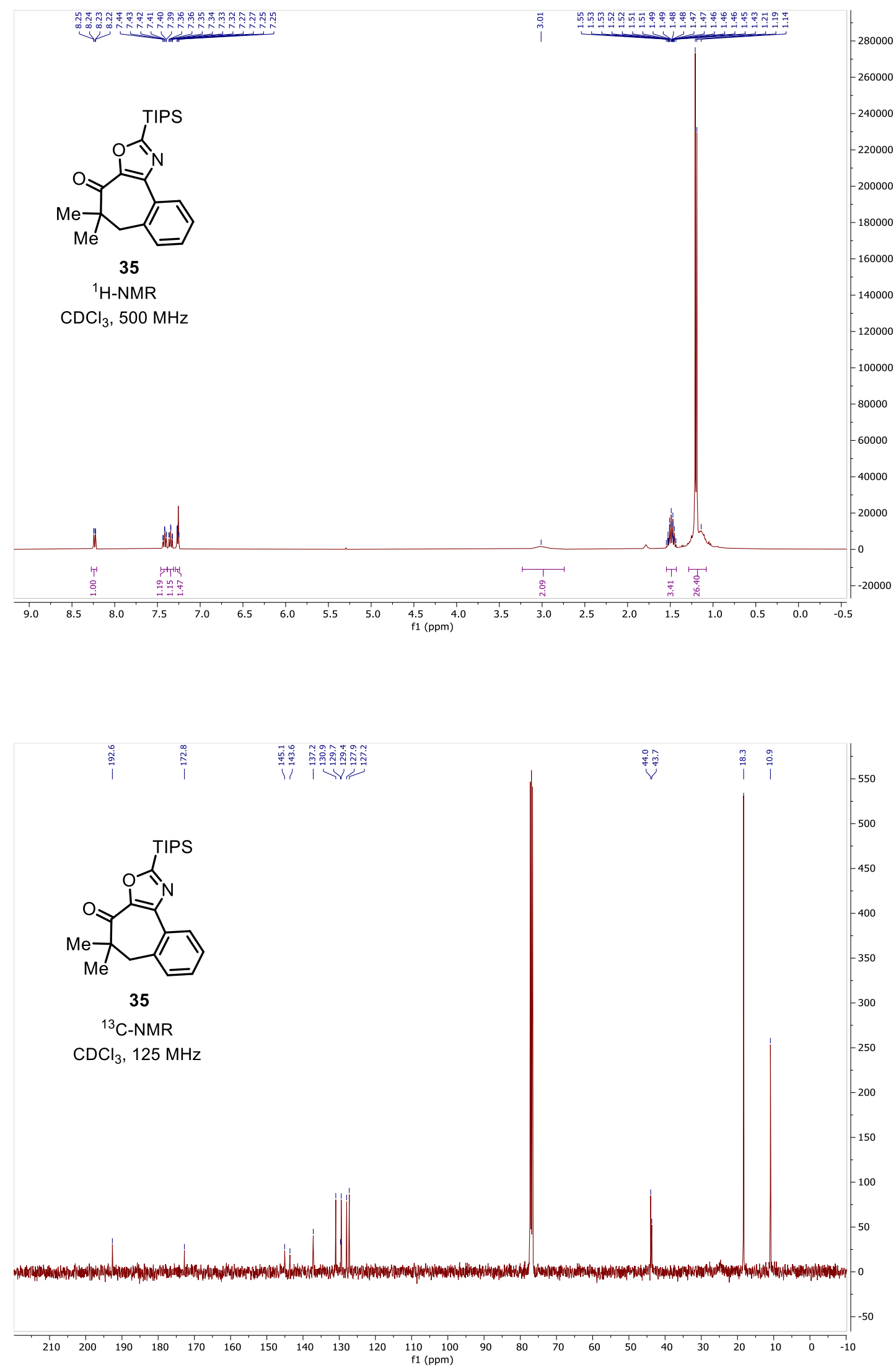

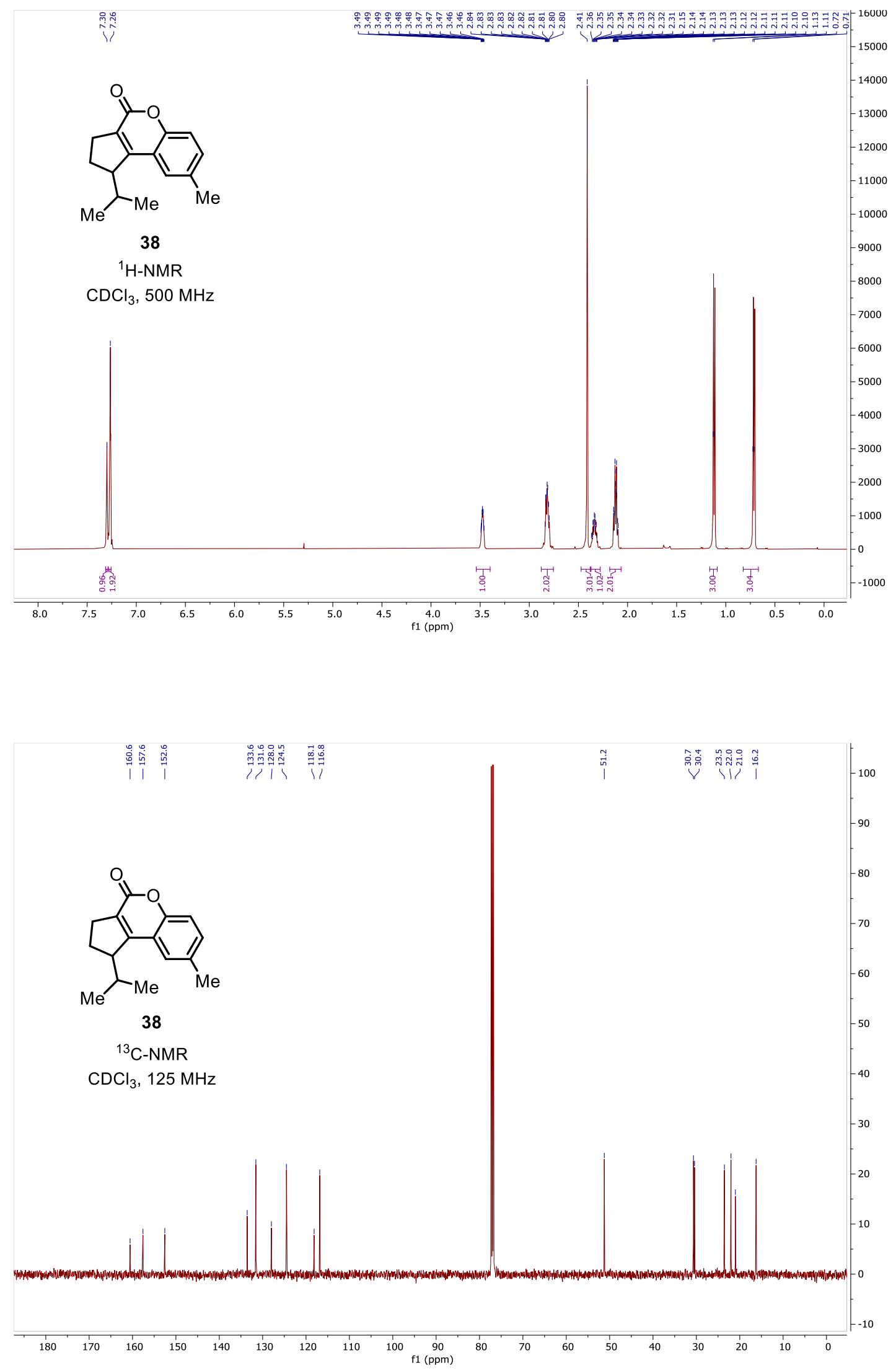

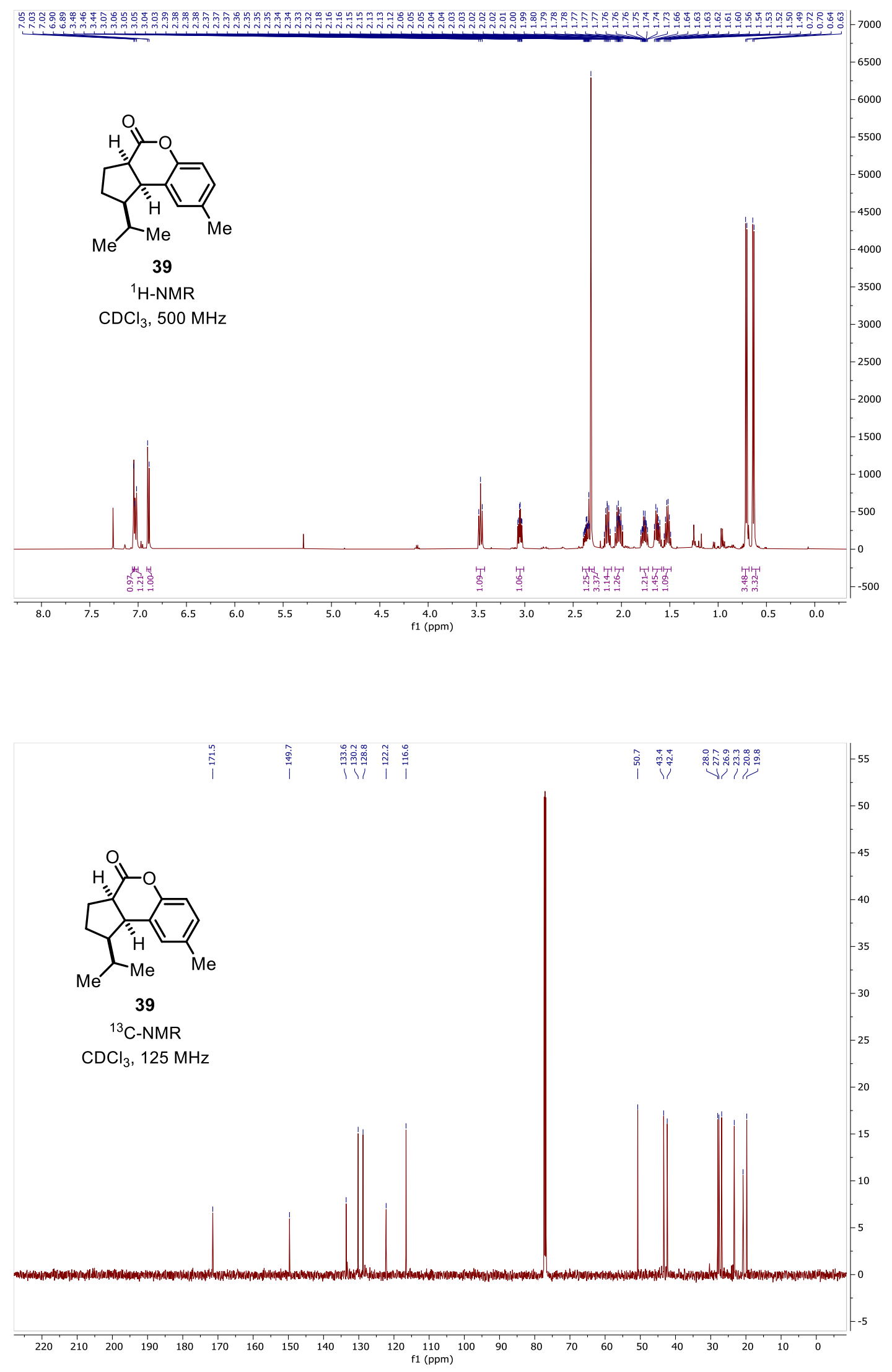

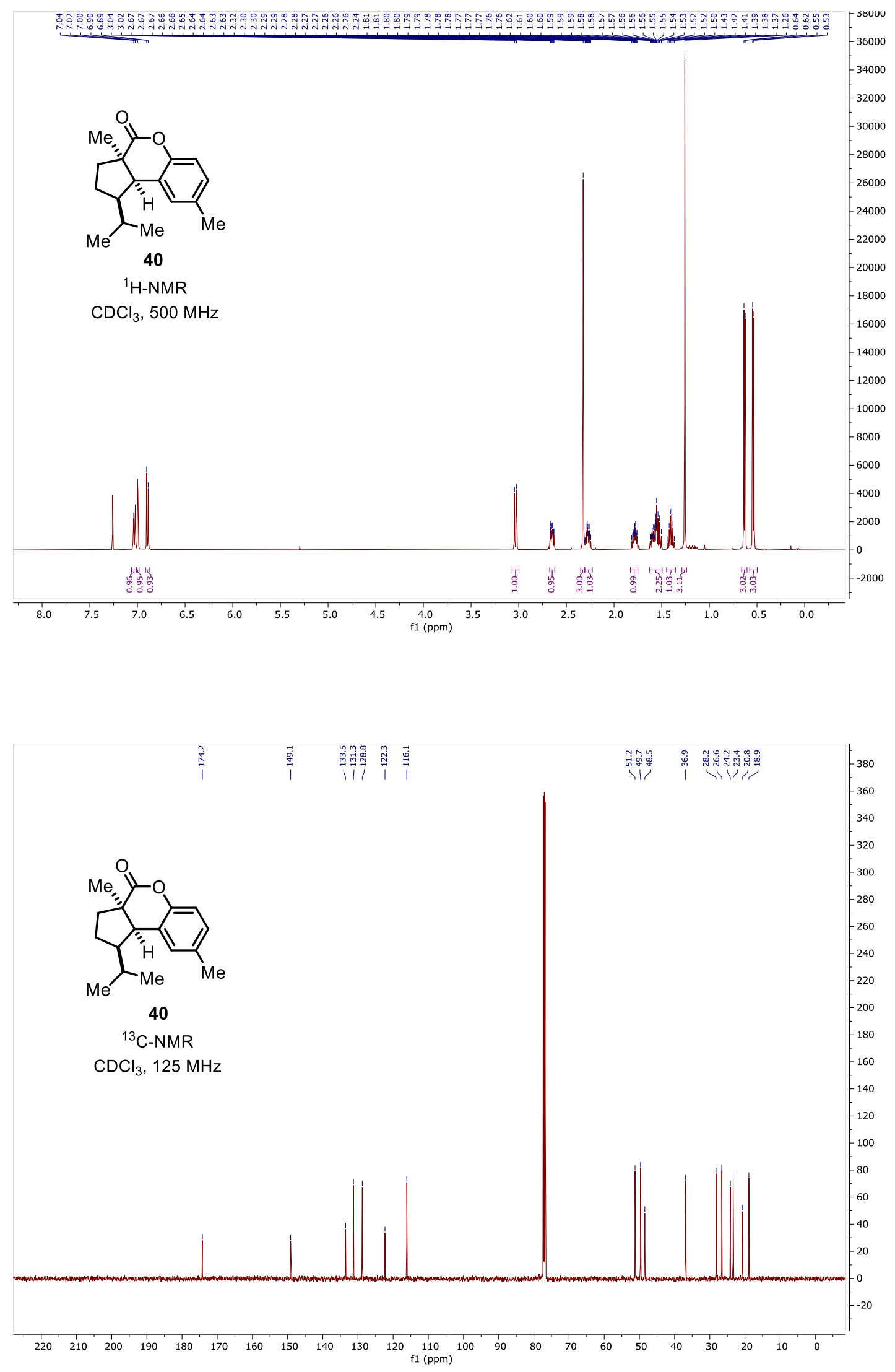

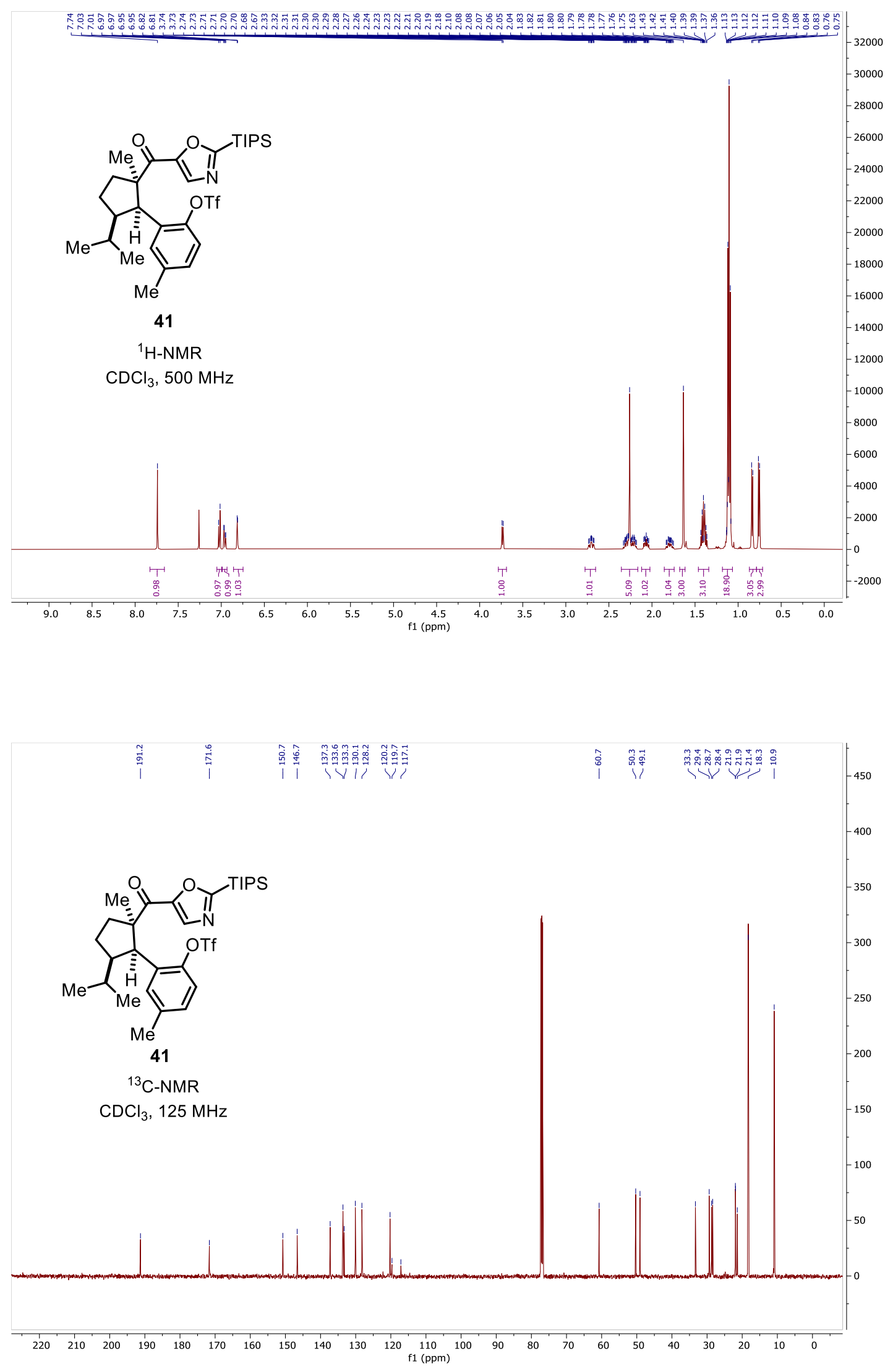


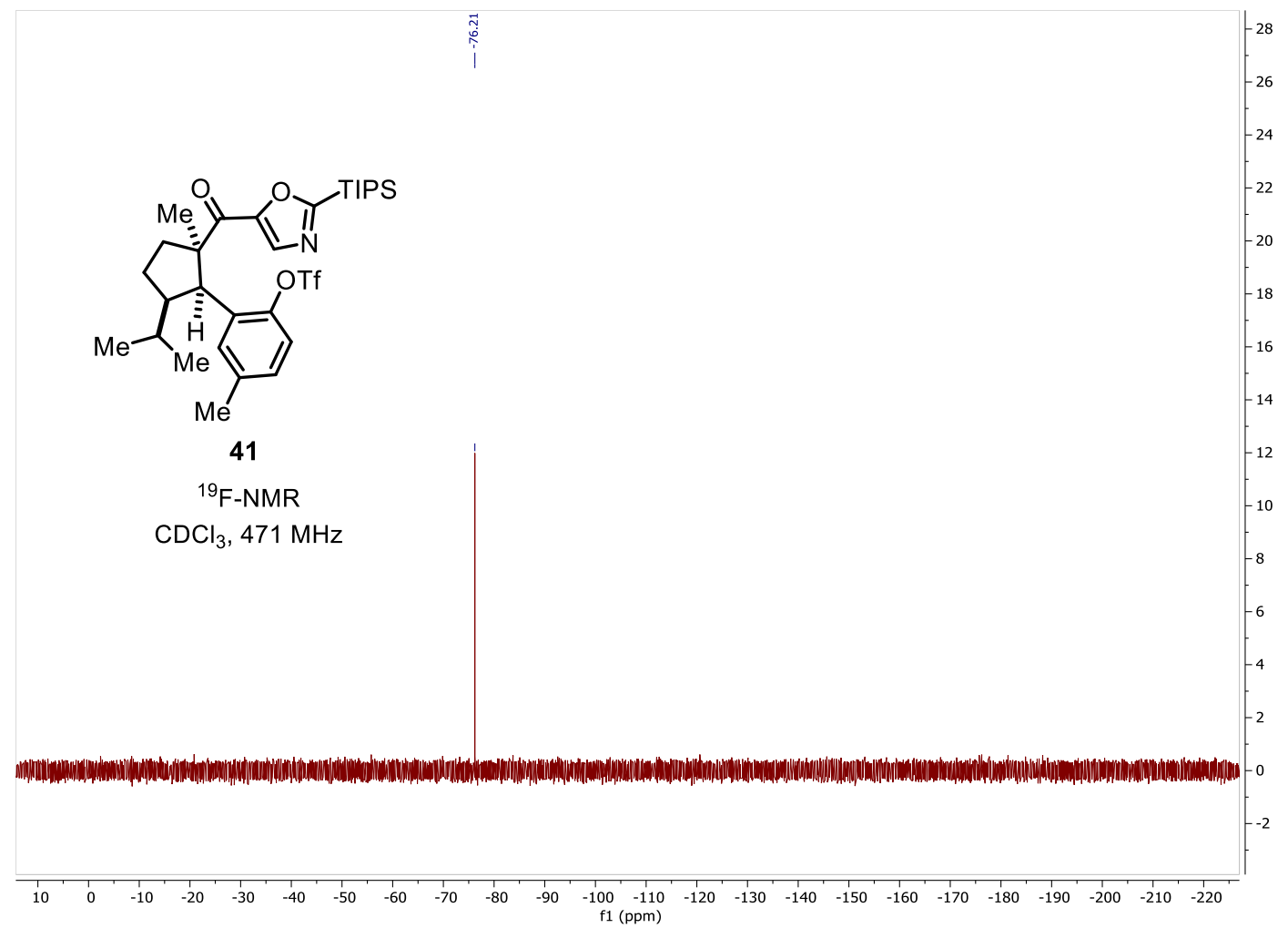



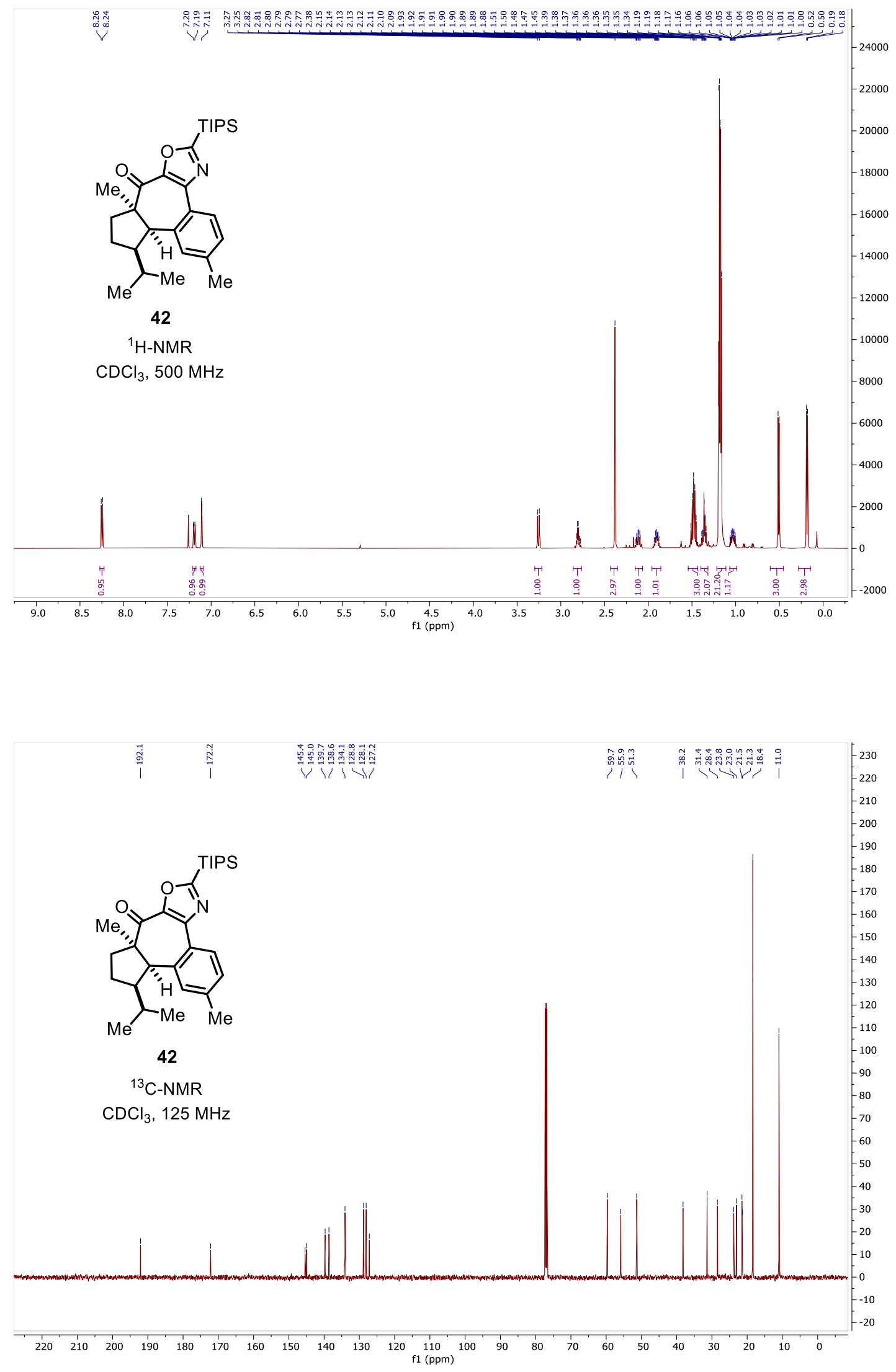

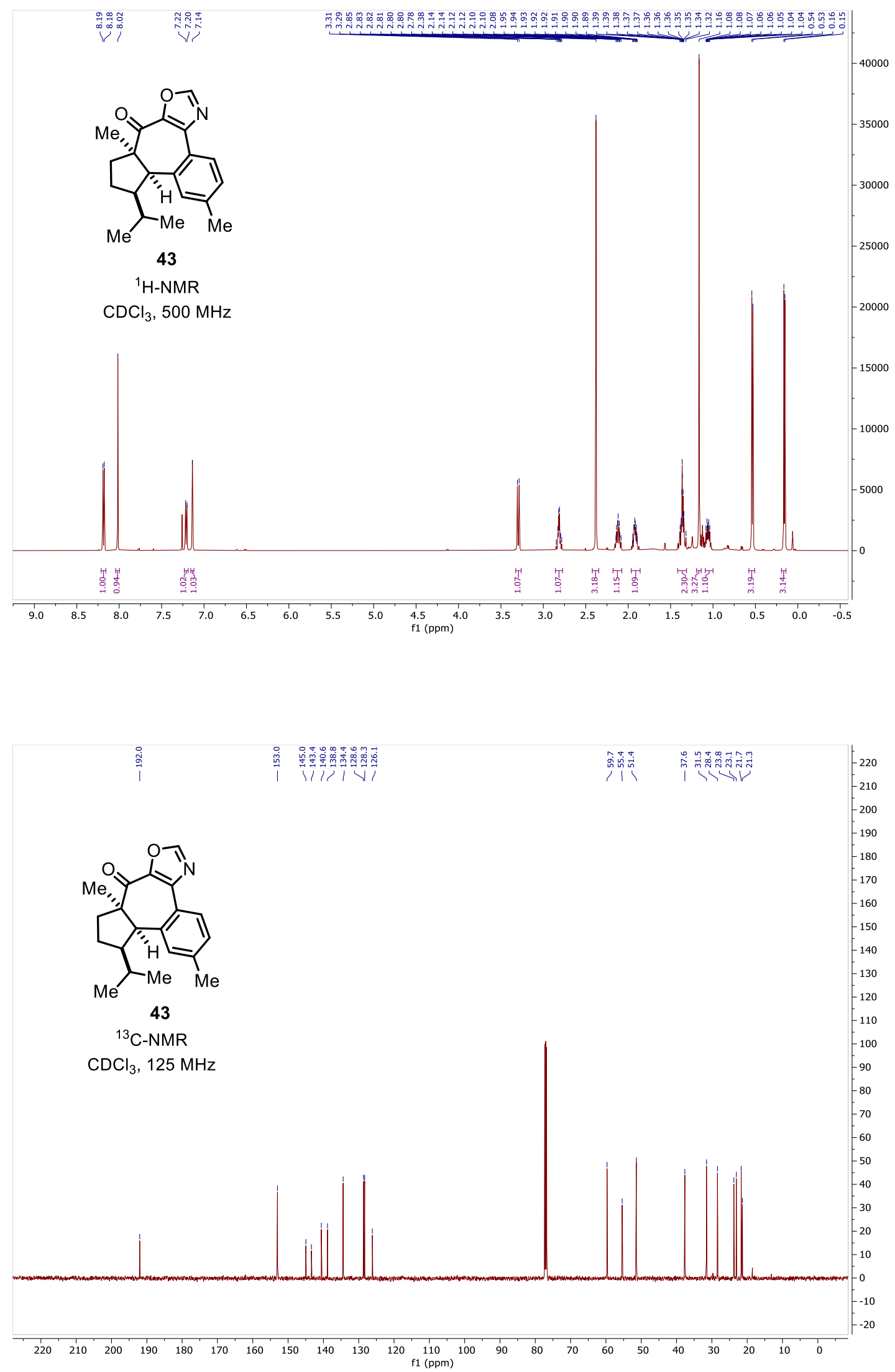

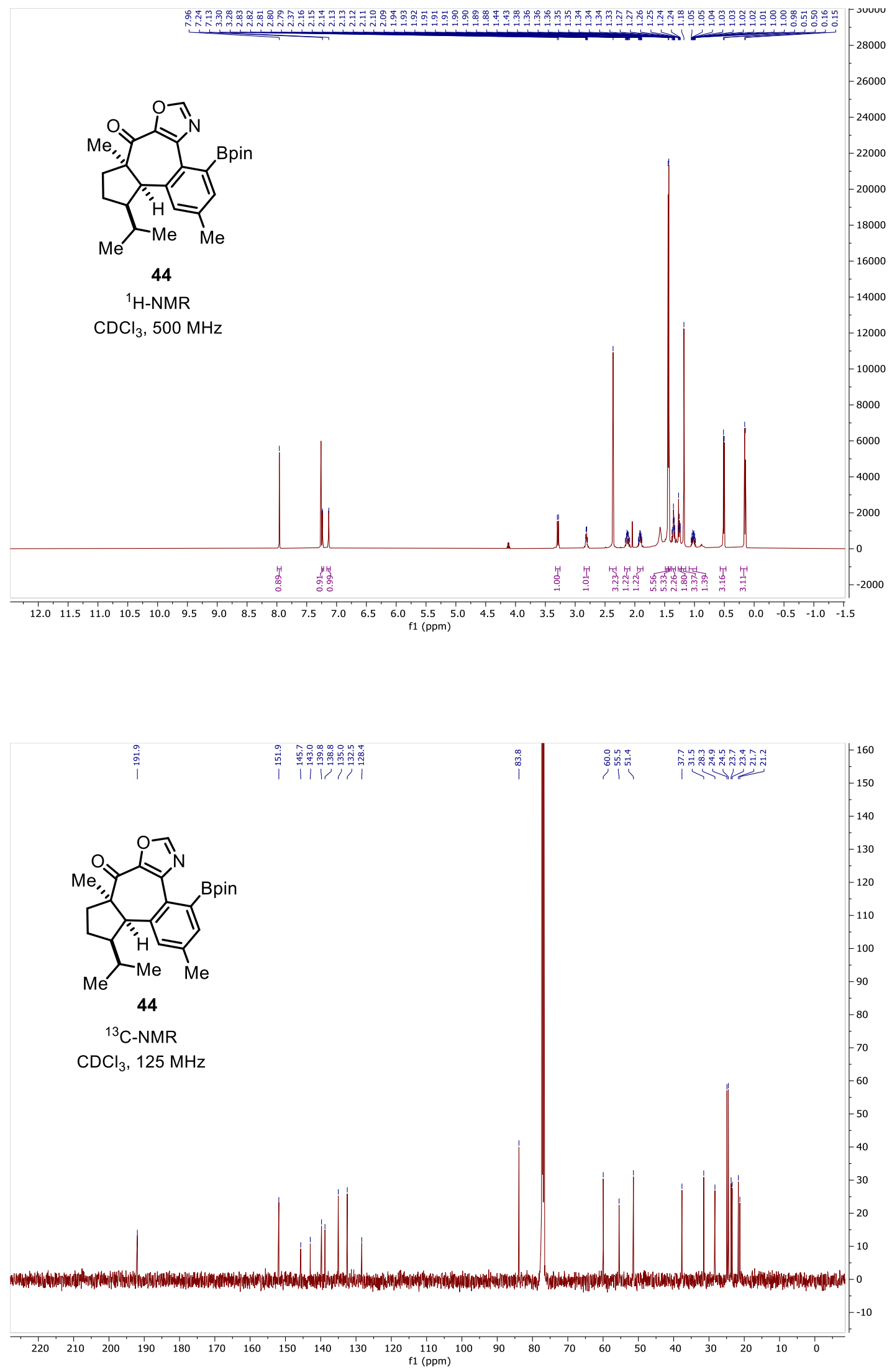

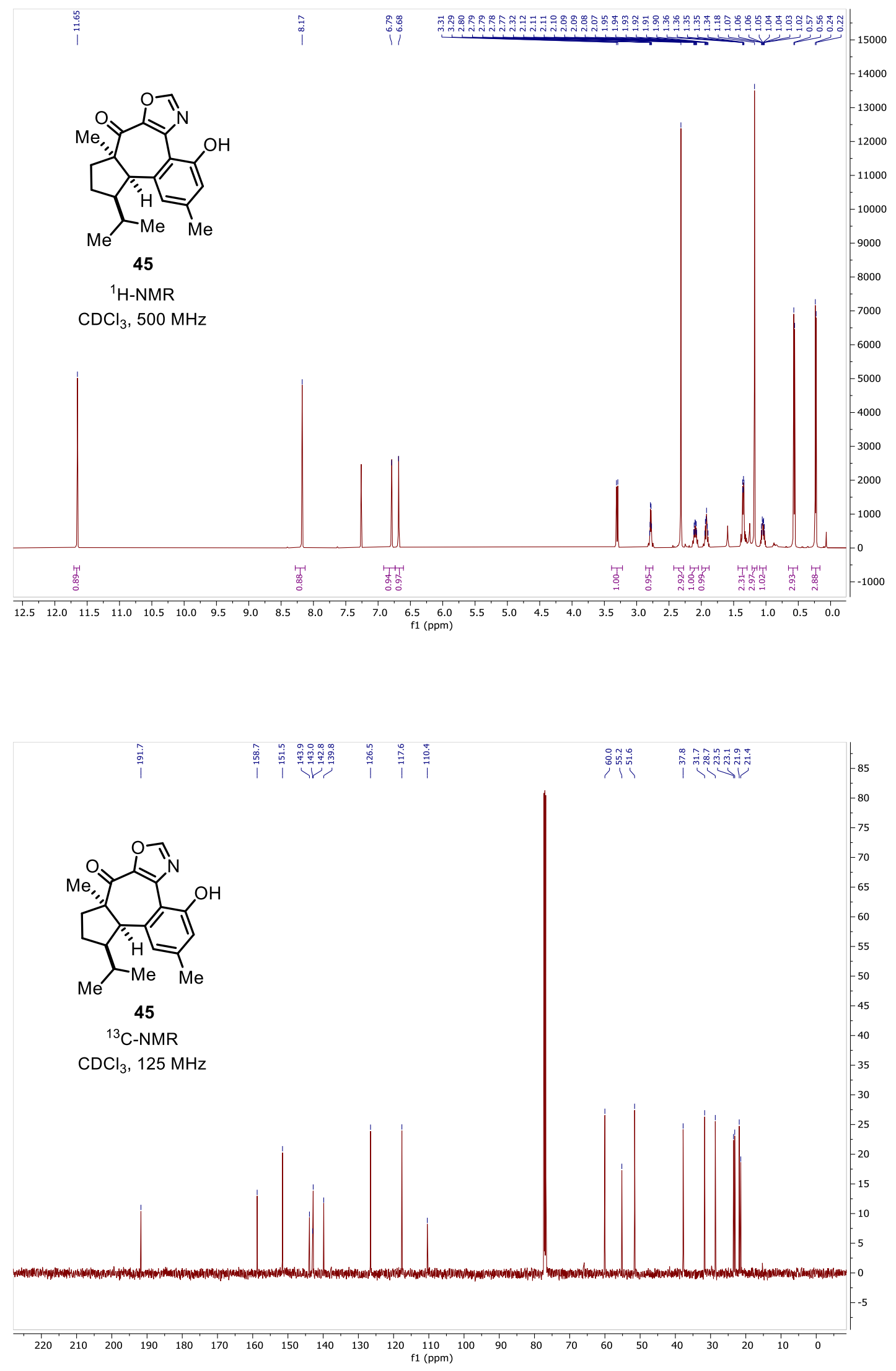

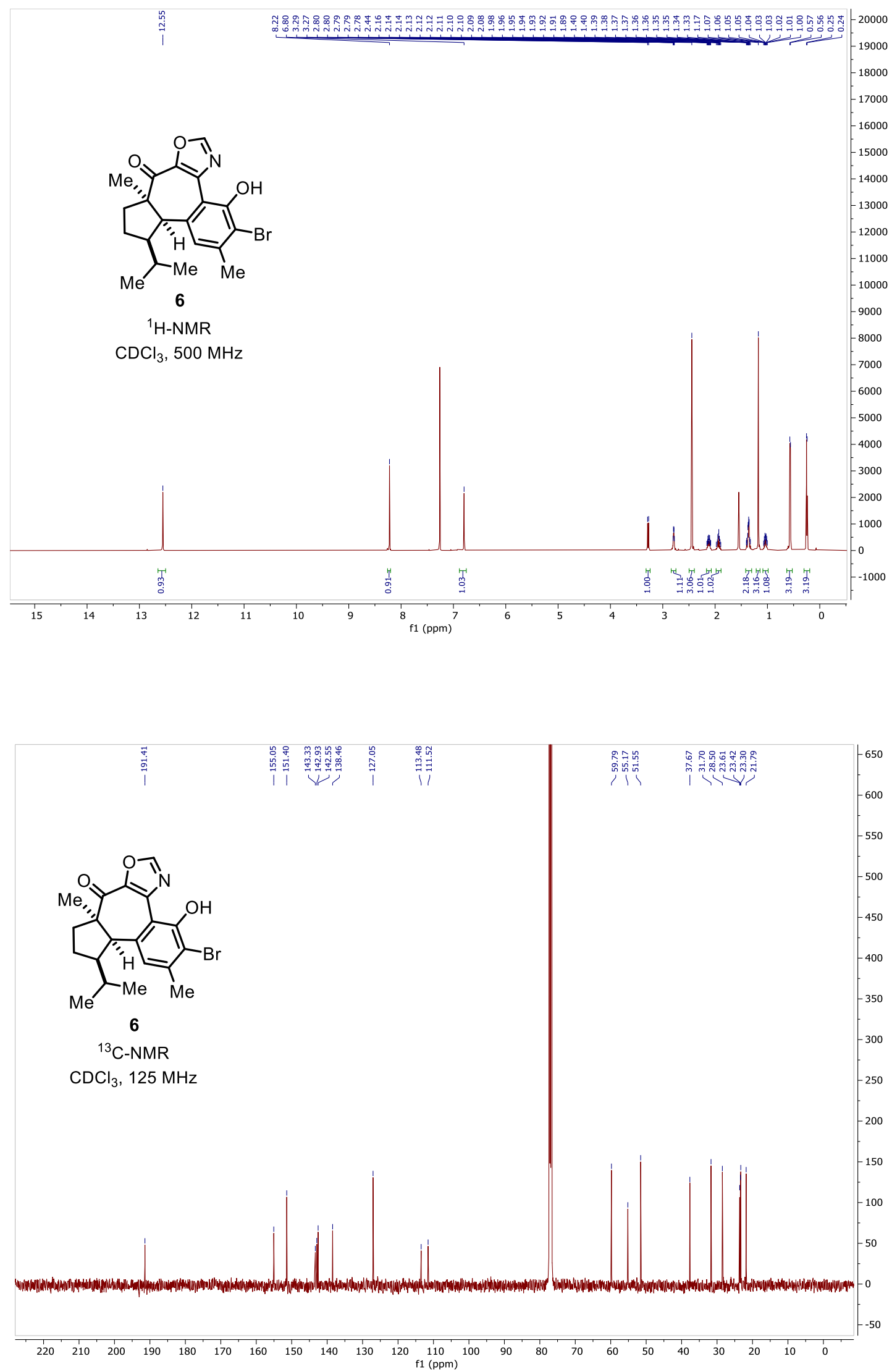

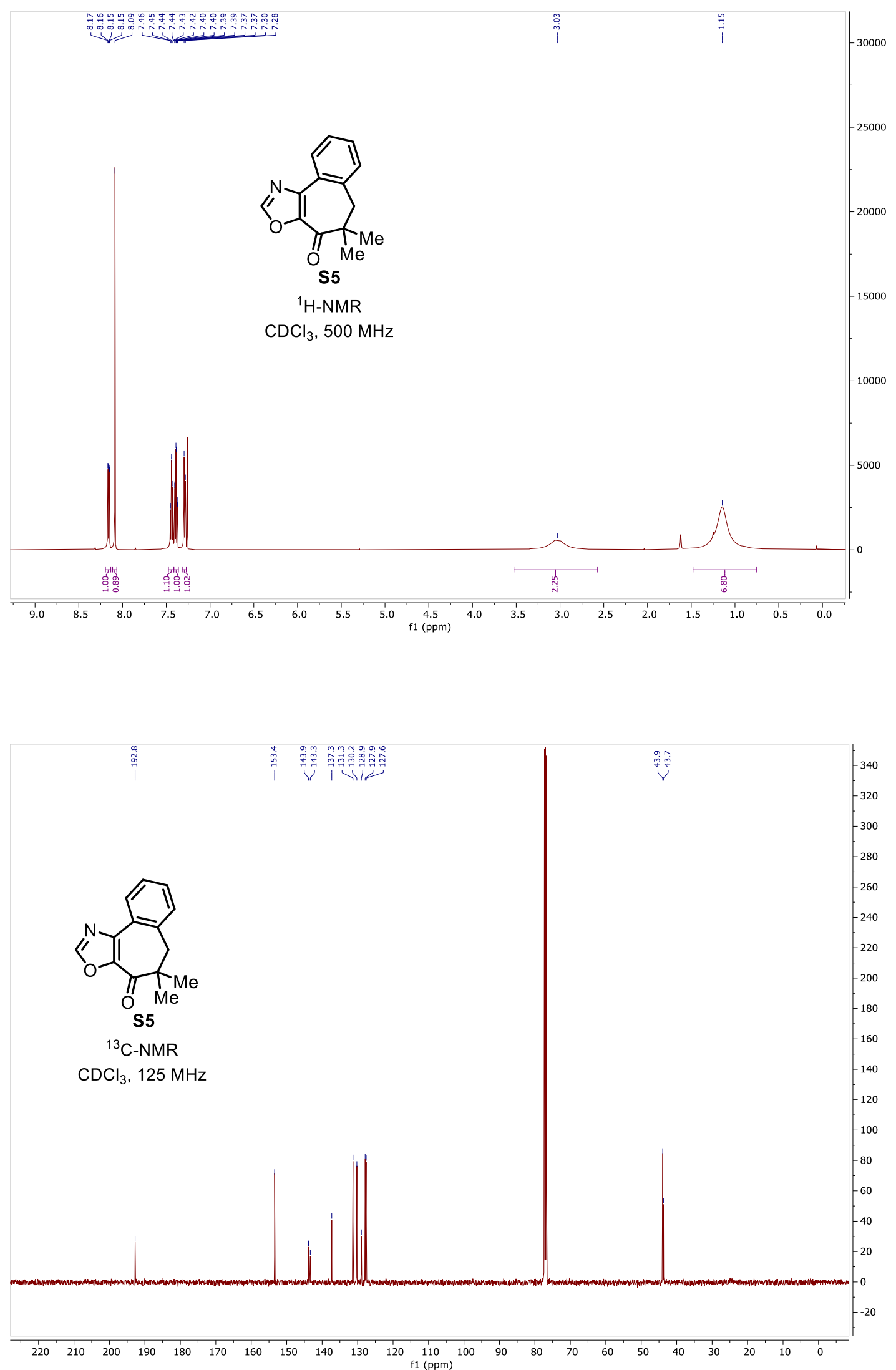